\title{
Inclusion problem in second gradient elasticity
}

\author{
Hansong $\mathrm{Ma}^{\mathrm{a}}$, Gengkai $\mathrm{Hu}^{\mathrm{b}}$, Yueguang Wei ${ }^{\mathrm{c}, *}$, Lihong Liang ${ }^{\mathrm{a}}$ \\ a State-Key Laboratory of Nonlinear Mechanics, Institute of Mechanics, Chinese Academy of Sciences, Beijing 100190, PR China \\ ${ }^{\mathrm{b}}$ School of Aerospace Engineering, Beijing Institute of Technology, Beijing 100081, PR China \\ ${ }^{\mathrm{c}}$ College of Engineering, Peking University, Beijing 100871, PR China
}

\section{A R T I C L E I N F O}

\section{Article history:}

Received 2 May 2018

Revised 28 June 2018

Accepted 18 July 2018

Available online 27 August 2018

\section{Keywords:}

Green's function

Eshelby tensor

Second gradient

Inclusion

Effective modulus

\begin{abstract}
A B S T R A C T
The Green's function and Eshelby tensors of an infinite linear isotropic second gradient continuum are derived for an inclusion of arbitrary shape. Particularly for spherical, cylindrical and ellipsoidal inclusions, Eshelby tensors and their volume averages are obtained in an analytical form. It is found that the Eshelby tensors are not uniform inside the inclusion even for a spherical inclusion, and their variations depend on the two characteristic lengths of second gradient theory. When size of inclusion is large enough compared to the characteristic lengths, the Eshelby tensor of the second gradient medium is reduced to the classical one, as expected. It is also demonstrated that the existing Green's functions and Eshelby tensors of couple stress theory, Aifantis, Kleinert and Wei-Hutchinson special strain gradient theories could be recovered as special cases. This work paves the way for constructing micromechanical method to predict size effect of composite materials, as shown for the effective modulus of particulate composite derived with the proposed theory.
\end{abstract}

(c) 2018 Elsevier Ltd. All rights reserved.

\section{Introduction}

Microcontinuum theory (Eringen, 1999) is considered as an efficient tool to characterize overall mechanics response for microstructured materials (Buechner \& Lakes, 2003; Chen, Lee, \& Eskandarian, 2004; Eringen, 1999). This high order theory incorporates the micro-deformation of microstructure inside of a material point in addition to translation of its inertia center. Depending on choice of micro-deformation mode, different simplified microcontinuum theories are proposed. For example, micromorphic theory (Eringen, 1999; Forest \& Sievert, 2003) assumes an arbitrary constant micro-deformation, it is the most general first grade microcontinuum theory. More specified theory can be developed by further assuming this constant micro-deformation, for example an independent rigid rotation, this gives micropolar theory (Eringen, 1968). For small deformation and slow motion assumption (Shat \& Abdelkefi, 2016), the micromorphic theory is consistent with microstructure theory of Mindlin (1964). Second gradient theory (Germain, 1973), which is also called strain gradient theory, is a special case of microstructure theory (Mindlin, 1964) by specifying the micro-deformation to be macro-displacementgradient. Therefore the theory has only the macro-displacement as degree of freedom and is easy to be implemented. There are several well-known simplified versions of second gradient theory, such as the couple stress theory (Koiter, 1964; Mindlin \& Tiersten, 1962; Toupin, 1962) corresponding to letting the micro-deformation gradient be only rotation gradient, Kleinert strain gradient theory (Kleinert, 1989) corresponding to considering the gradient of volumetric strain in addition to rotation

\footnotetext{
* Corresponding author.

E-mail addresses: mahs@Inm.imech.ac.cn (H. Ma), weiyg@pku.edu.cn (Y. Wei).
} 
gradient as the micro-deformation gradient, etc. Some other models, like Aifantis (Altan \& Aifantis, 1997; Gao \& Park, 2007) and Wei \& Hutchinson's strain gradient elasticity theory (Song, Liu, Ma, Liang, \& Wei, 2014; Wei, 2006; Wei \& Hutchinson, 1997), use strain gradient or second gradient of displacement as the micro-deformation gradient and define different constitutive relations. The objective of developing these high order continuum theories is to characterize the size effect well observed when size of structure is decreasing to micro or nano scale (Fleck, Muller, Ashby, \& Hutchinson, 1994; Kouzeli \& Mortensen, 2002), the microstructure comes into play in this case which is unable to be accounted for by Cauchy continuum theory without microstructure (Hu, Liu, \& Lu, 2005).

In order to predict the size effect manifested in composites materials, proper homogenization method should be established. Inclusion problem is an essential step to build micromechanical models, in which Eshelby tensor is a key factor. Eshelby tensors in some microcontinuum models have already been obtained. For example, Eshelby tensors of spherical, cylindrical inclusions (Cheng \& He, 1995, 1997) and ellipsoidal inclusion (Ma \& Hu, 2006) are derived for micropolar medium and Aifantis strain gradient medium (Gao \& Ma, 2009, 2010; Ma \& Gao, 2010; Zheng \& Zhao, 2004) derived Eshelby tensor for spherical inclusion in couple stress medium. Zhang and Sharma (2005) examined Eshelby tensor of Kleinert's strain gradient theory. Unlike the classical Eshelby tensor, these Eshelby tensors are not uniform inside the inclusion domain. But based on the average of these Eshelby tensors, an average equivalent inclusion method could be established for composites (Liu \& Hu, 2005; Ma \& Gao, 2014; Sharma \& Dasgupta, 2002; Xun, Hu, \& Huang, 2004), and it can be used to predict the size-dependence of inclusion on overall elastoplastic property of composites.

As discussed above, the second gradient theory is a more general high order theory and easy to use, it may offer an alternative and flexible tool to establish homogenization method for composites. However inclusion problem of a general isotropic second gradient medium has not been addressed yet, which is the objective of this manuscript. The Green's function and Eshelby tensor will be derived in analytical form, and their interconnections with the existing strain gradient theories, couple stress, Aifantis, Kleinert, and Wei \& Hutchinson's models will be demonstrated. Finally effective modulus of a particulate composite will be presented to illustrate the capacity to predict size effect. The manuscript is arranged as follows, in Section 2, Green's function of a general isotropic second gradient medium will be derived. The inclusion problem will be examined in Section 3, and its connection with different strain gradient theories will be discussed in Section 4. Some examples will be presented in Section 5 to illustrate characteristic of derived Eshelby tensor. In Section 6, effective modulus of a composite with spherical inclusion will be given to characterize size effect of mechanical behavior. Finally some conclusions are presented.

\section{Green's function}

For a linear isotropic second gradient continuum, the governing equations are (Mindlin, 1964): geometrical relations:

$$
\varepsilon_{i j}=\frac{1}{2}\left(u_{i, j}+u_{j, i}\right)=\varepsilon_{j i}, \quad \eta_{i j k}=\nabla \nabla \mathbf{u}=u_{k, i j}=\eta_{j i k}
$$

equilibrium equation:

$$
\sigma_{i k, i}-\tau_{i j k, i j}+f_{k}=0
$$

constitutive equations:

$$
\sigma_{i j}=\frac{\partial W}{\partial \varepsilon_{i j}}=C_{i j k l} \varepsilon_{k l} \quad \tau_{i j k}=\frac{\partial W}{\partial \eta_{i j k}}=T_{i j k l m n} \eta_{l m n}
$$

where $u_{i}$ is displacement vector, $\varepsilon_{i j}$ and $\eta_{i j k}$ are strain and strain gradient tensors, $\sigma_{i j}$ and $\tau_{i j k}$ are stress and high-order stress tensors. $f_{k}$ is body force vector. $W$ is strain energy density function, its expression is (Mindlin, 1965):

$$
W=\frac{1}{2} \lambda \varepsilon_{i i} \varepsilon_{j j}+\mu \varepsilon_{i j} \varepsilon_{i j}+a_{1} \eta_{i j j} \eta_{i k k}+a_{2} \eta_{i i k} \eta_{k j j}+a_{3} \eta_{i i k} \eta_{j j k}+a_{4} \eta_{i j k} \eta_{i j k}+a_{5} \eta_{i j k} \eta_{k j i}
$$

where $\lambda, \mu$ are Lame constants, $a_{1}, a_{2}, a_{3}, a_{4}, a_{5}$ are additional constants introduced in second gradient theory. $C_{i j k l}$ and $T_{i j k l m n}$ are elasticity tensors of second gradient materials, their expressions are:

$$
\begin{aligned}
C_{i j k l}=\lambda & \delta_{i j} \delta_{k l}+\mu \delta_{i k} \delta_{j l}+\mu \delta_{i l} \delta_{j k} \\
T_{i j k l m n}= & \frac{a_{1}}{2}\left(\delta_{i l} \delta_{j k} \delta_{m n}+\delta_{i m} \delta_{j k} \delta_{l n}+\delta_{i k} \delta_{j m} \delta_{l n}+\delta_{i k} \delta_{j l} \delta_{m n}\right) \\
& +\frac{a_{2}}{2}\left(\delta_{i j} \delta_{l k} \delta_{m n}+\delta_{i j} \delta_{m k} \delta_{l n}+\delta_{i n} \delta_{j k} \delta_{l m}+\delta_{i k} \delta_{j n} \delta_{m l}\right)+2 a_{3} \delta_{i j} \delta_{k n} \delta_{l m} \\
& +a_{4}\left(\delta_{i m} \delta_{j l} \delta_{k n}+\delta_{i l} \delta_{j m} \delta_{k n}\right)+\frac{a_{5}}{2}\left(\delta_{i n} \delta_{j m} \delta_{k l}+\delta_{i n} \delta_{j l} \delta_{k m}+\delta_{i m} \delta_{j n} \delta_{l k}+\delta_{i l} \delta_{j n} \delta_{m k}\right)
\end{aligned}
$$

$\delta_{i j}$ is Kronecker delta. 
Substituting geometrical Eq. (2.1) and constitutive Eq. (2.3) to equilibrium Eq. (2.2), the displacement equilibrium equation is obtained:

$$
(\lambda+\mu) u_{j, j i}+\mu u_{i, k k}-\left[(\lambda+2 \mu) l_{1}^{2} u_{j, j i}-\mu l_{2}^{2}\left(u_{j, j i}-u_{i, k k}\right)\right]_{, l l}+f_{i}=0
$$

or in vector presentation:

$$
(\lambda+2 \mu)\left(1-l_{1}^{2} \nabla^{2}\right) \nabla \nabla \cdot \mathbf{u}-\mu\left(1-l_{2}^{2} \nabla^{2}\right) \nabla \times \nabla \times \mathbf{u}+\mathbf{f}=0
$$

where

$$
l_{1}^{2}=\frac{2\left(a_{1}+a_{2}+a_{3}+a_{4}+a_{5}\right)}{\lambda+2 \mu}, \quad l_{2}^{2}=\frac{2\left(a_{3}+a_{4}\right)}{\mu}
$$

$l_{1}, l_{2}$ are two material characteristic lengths of second gradient theory.

To derive fundamental solution of Eq. (2.7), the method of Sandru (1966) is used and following differential operators are defined:

$$
\begin{aligned}
& \square_{1}=(\lambda+2 \mu)\left(1-l_{1}^{2} \Delta\right) \Delta, \quad \square_{2}=(\lambda+2 \mu)\left(1-l_{1}^{2} \Delta\right)-\mu\left(1-l_{2}^{2} \Delta\right) \\
& \square_{3}=\mu\left(1-l_{2}^{2} \Delta\right) \Delta, \quad \text { and } \square_{1}=\square_{2} \Delta+\square_{3}
\end{aligned}
$$

The body force vector $\mathbf{f}$ can be decomposed into scalar and vector potentials as:

$$
\mathbf{f}=\nabla \Pi_{0}+\nabla \times \boldsymbol{\Pi}
$$

where $\Pi_{0}$ is a scalar potential, $\Pi$ is a vector potential, and $\nabla \cdot \Pi=0$.

It can be proved that if the following relations are satisfied,

$$
\square_{1} \Lambda_{0}=-\Pi_{0}, \square_{3} \Lambda=-\Pi \text { and } \nabla \cdot \Lambda=0
$$

solution of Eq. (2.7) is:

$$
\mathbf{u}=\nabla \Lambda_{0}+\nabla \times \boldsymbol{\Lambda}
$$

The detailed proof is listed in Appendix A.

Let $\mathbf{Q}$ be an impulse body load applied at position $\mathbf{x}^{\prime}$ in an infinitely extended body, that is:

$$
\mathbf{f}=\mathbf{Q} \delta\left(\mathbf{x}-\mathbf{x}^{\prime}\right)
$$

where $\delta\left(\mathbf{x}-\mathbf{x}^{\prime}\right)$ is Dirac delta function.

Following Sandru (1966), when $\mathbf{f}=\nabla \Pi_{0}+\nabla \times \Pi$, we have:

$$
\begin{aligned}
\Pi_{0} & =-\frac{1}{4 \pi} \mathbf{Q} \cdot \nabla\left(\frac{1}{r}\right) \\
\boldsymbol{\Pi} & =-\frac{1}{4 \pi} \mathbf{Q} \times \nabla\left(\frac{1}{r}\right)
\end{aligned}
$$

where $r=\left|\mathbf{x}-\mathbf{x}^{\prime}\right|$.

By suing Fourier transformation and inverse transformation, solutions of Eq. (2.11) are obtained:

$$
\begin{aligned}
\Lambda_{0} & =\frac{1}{8 \pi(\lambda+2 \mu)} \mathbf{Q} \cdot \nabla r+\frac{l_{1}^{2}}{4 \pi(\lambda+2 \mu)} \mathbf{Q} \cdot \nabla\left(\frac{1-e^{-r / l_{1}}}{r}\right) \\
\mathbf{\Lambda} & =-\frac{l_{2}^{2}}{4 \pi \mu} \nabla \times \mathbf{Q}\left(\frac{1-e^{-r / l_{2}}}{r}\right)-\frac{1}{8 \pi \mu} \nabla \times(\mathbf{Q} r)
\end{aligned}
$$

According to Eq. (2.12), the fundamental displacement field of second gradient continuum is:

$$
\begin{aligned}
\mathbf{u}= & \frac{1}{8 \pi \mu}\left(\frac{2}{r} \mathbf{Q}-\frac{\lambda+\mu}{\lambda+2 \mu} \mathbf{Q} \cdot \nabla \nabla r\right)+\frac{l_{1}^{2}}{4 \pi(\lambda+2 \mu)} \mathbf{Q} \cdot \nabla \nabla\left(\frac{1-e^{-r / l_{1}}}{r}\right) \\
& -\frac{l_{2}^{2}}{4 \pi \mu} \mathbf{Q} \cdot \nabla \nabla\left(\frac{1-e^{-r / l_{2}}}{r}\right)-\frac{1}{4 \pi \mu} \mathbf{Q} \frac{e^{-r / l_{2}}}{r}
\end{aligned}
$$

or written in vector component presentation:

$$
u_{i}=G_{i j} Q_{j}=\left(G_{i j}^{c}+G_{i j}^{1}+G_{i j}^{2}\right) Q_{j}
$$

where $G_{i j}^{c}=\frac{1}{8 \pi \mu}\left(\frac{2}{r} \delta_{i j}-\frac{\lambda+\mu}{\lambda+2 \mu} r_{, i j}\right)$

$$
G_{i j}^{1}=\frac{l_{1}^{2}}{4 \pi(\lambda+2 \mu)}\left(\frac{1-e^{-r / l_{1}}}{r}\right)_{, i j}
$$




$$
G_{i j}^{2}=-\frac{l_{2}^{2}}{4 \pi \mu}\left(\frac{1-e^{-r / l_{2}}}{r}\right)_{, i j}-\frac{1}{4 \pi \mu} \frac{e^{-r / l_{2}}}{r} \delta_{i j}
$$

$G_{i j}$ is Green's function of second gradient theory. $G_{i j}^{c}$ is the classical Green's function, $G_{i j}^{1}$ and $G_{i j}^{2}$ are the ones related respectively to the characteristic length $l_{1}$ and $l_{2}$ of second gradient theory.

\section{Inclusion problem}

\subsection{Eigen deformation problem}

Consider an infinite linear isotropic second gradient material with eigenstrain $\varepsilon_{i j}^{*}$ and eigenstrain-gradient $\eta_{i j k}^{*}$, which are nonelastic deformations (Mura, 1987). The constitutive Eq. (2.3) must be modified to:

$$
\sigma_{i j}=C_{i j k l}\left(\varepsilon_{k l}-\varepsilon_{k l}^{*}\right) \quad \tau_{i j k}=T_{i j k l m n}\left(\eta_{l m n}-\eta_{l m n}^{*}\right)
$$

Substituting Eq. (2.1) and Eq. (3.1) to Eq. (2.2) and ignoring body force, we obtain:

$$
\begin{aligned}
& \frac{1}{2} C_{i k m n}\left(u_{n, m i}+u_{m, n i}\right)-T_{i j k l m n} u_{n, l m i j}+f_{k}^{*}=0 \\
& \text { where }: f_{k}^{*}=-\left(C_{i k m n} \varepsilon_{m n, i}^{*}-T_{i j k l m n} \eta_{l m n, i j}^{*}\right)
\end{aligned}
$$

So the eigendeformation problem is transformed to an equivalent distributed body force problem.

To derive the displacement $u_{i}$ in terms of the obtained Green's function, we employ the work reciprocal theorem of second gradient medium:

$$
\int_{V} f_{k} u_{k}^{\prime} d \mathbf{x}=\int_{V} f^{\prime}{ }_{k} u_{k} d \mathbf{x}
$$

where the quantities with and without a prime are two distinct independent sets of load and resulted displacement fields.

Let $f_{k}^{\prime}=\delta_{k i} \delta\left(\mathbf{x}-\mathbf{x}^{\prime}\right)$, then $u_{k}^{\prime}=G_{k i}^{c}+G_{k i}^{1}+G_{k i}^{1}=G_{k i}$

Using Eq. (3.4) and property of Dirac function, we obtain:

$$
u_{i}(\mathbf{x})=\int_{V} f_{k} G_{k i}\left(\mathbf{x}-\mathbf{x}^{\prime}\right) d \mathbf{x}^{\prime}
$$

By substituting body force Eq. (3.3) into Eq. (3.5), displacement field due to previously prescribed eigendeformation can be obtained:

$$
u_{s}(\mathbf{x})=-\int_{V} C_{i k m n} \varepsilon_{m n}^{*} G_{k s, i}\left(\mathbf{x}-\mathbf{x}^{\prime}\right) d \mathbf{x}^{\prime}+\int_{V} T_{i j k l m n} \eta_{l m n}^{*} G_{k s, i j}\left(\mathbf{x}-\mathbf{x}^{\prime}\right) d \mathbf{x}^{\prime}
$$

Eq. (3.6) is a general expression and valid for any eigendeformation. With help of Eqs. (2.17), (2.18), (2.1), (2.3), local elastic displacement, strain and stress fields due to any eigendeformation can be obtained completely.

\subsection{Eshelby tensors}

Considering an inclusion $\Omega$ in an infinite linear isotropic second gradient material, uniform eigenstrain $\varepsilon_{i j}^{*}$ and eigenstrain-gradient $\eta_{i j k}^{*}$ are prescribed in the inclusion. And eigendeformation is zero outside of $\Omega$. Here the inclusion $\Omega$ could be an arbitrary shape.

Then Eq. (3.6) can be rewritten as:

$$
\begin{aligned}
& u_{s}(\mathbf{x})=I_{s m n}(\mathbf{x}) \varepsilon_{m n}^{*}+J_{s l m n}(\mathbf{x}) \eta_{l m n}^{*} \\
& \text { where : } \quad I_{s m n}(\mathbf{x})=-C_{i k m n} \int_{\Omega} G_{k s, i} d \mathbf{x}^{\prime}, \quad J_{s l m n}(\mathbf{x})=T_{i j k l m n} \int_{\Omega} G_{k s, i j} d \mathbf{x}^{\prime}
\end{aligned}
$$

By differentiating both sides of Eq. (3.7) and according to geometrical equation, the strain and strain gradient in $\Omega$ induced by the prescribed eigenstrain and eigenstrain-gradient can be written as:

$$
\varepsilon_{s t}(\mathbf{x})=S_{s t m n}(\mathbf{x}) \varepsilon_{m n}^{*}+\hat{S}_{s t l m n}(\mathbf{x}) \eta_{l m n}^{*}, \quad \eta_{r t s}(\mathbf{x})=N_{r t s m n}(\mathbf{x}) \varepsilon_{m n}^{*}+\hat{N}_{r t s l m n}(\mathbf{x}) \eta_{l m n}^{*}
$$

where:

$$
\begin{aligned}
S_{s t m n}(\mathbf{x}) & =\frac{1}{2}\left[I_{t m n, s}(\mathbf{x})+I_{s m n, t}(\mathbf{x})\right], \hat{S}_{s t l m n}(\mathbf{x})=\frac{1}{2}\left[J_{t l m n, s}(\mathbf{x})+J_{s l m n, t}(\mathbf{x})\right] \\
N_{r t s m n}(\mathbf{x}) & =I_{s m n, r t}(\mathbf{x}), \hat{N}_{r t s l m n}(\mathbf{x})=J_{s l m n, r t}(\mathbf{x})
\end{aligned}
$$


and

$$
\begin{aligned}
I_{s m n}(\mathbf{x})= & \frac{\lambda+\mu}{\lambda+2 \mu} \psi_{, s m n}(\mathbf{x})-\frac{\lambda}{\lambda+2 \mu} \delta_{m n} \phi_{, s}(\mathbf{x})-\delta_{s n} \phi_{, m}(\mathbf{x})-\delta_{m s} \phi_{, n}(\mathbf{x}) \\
& +\frac{\lambda}{\lambda+2 \mu} \delta_{m n} M_{, s}\left(\mathbf{x}, l_{1}\right)-\frac{2 \mu}{\lambda+2 \mu} l_{1}^{2}\left[\phi_{, s m n}(\mathbf{x})-M_{, s m n}\left(\mathbf{x}, l_{1}\right)\right] \\
& +2 l_{2}^{2}\left[\phi_{, s m n}(\mathbf{x})-M_{, s m n}\left(\mathbf{x}, l_{2}\right)\right]+\delta_{s n} M_{, m}\left(\mathbf{x}, l_{2}\right)+\delta_{s m} M_{, n}\left(\mathbf{x}, l_{2}\right) \\
J_{s l m n}(\mathbf{x})= & -\frac{(\lambda+\mu)\left(a_{4}+a_{5}\right)}{(\lambda+2 \mu) \mu} \psi_{, s l m n}(\mathbf{x})+\frac{2 \mu a_{1}-(\lambda+\mu) a_{2}}{2(\lambda+2 \mu) \mu}\left[\delta_{m n} \phi_{, s l}(\mathbf{x})+\delta_{l n} \phi_{, s m}(\mathbf{x})\right] \\
& +\frac{\mu a_{2}-2(\lambda+\mu) a_{3}}{(\lambda+2 \mu) \mu} \delta_{m l} \phi_{, s n}(\mathbf{x})+\frac{2 a_{4}}{\mu} \delta_{s n} \phi_{, m l}(\mathbf{x})+\frac{2 a_{5}}{\mu}\left[\delta_{s l} \phi_{, m n}(\mathbf{x})+\delta_{s m} \phi_{, l n}(\mathbf{x})\right] \\
& +\frac{2\left(a_{4}+a_{5}\right)}{(\lambda+2 \mu)} l_{1}^{2}\left[\phi_{, s l m n}(\mathbf{x})-M_{, s l m n}\left(\mathbf{x}, l_{1}\right)\right]-\frac{2 a_{1}+a_{2}}{2(\lambda+2 \mu)}\left[\delta_{m n} M_{, s l}\left(\mathbf{x}, l_{1}\right)+\delta_{l n} M_{, s m}\left(\mathbf{x}, l_{1}\right)\right] \\
& -\frac{2 a_{3}+a_{2}}{(\lambda+2 \mu)} \delta_{m l} M_{, s n}\left(\mathbf{x}, l_{1}\right)-\frac{2\left(a_{4}+a_{5}\right)}{\mu} l_{2}^{2}\left[\phi_{, s l m n}(\mathbf{x})-M_{s l m n}\left(\mathbf{x}, l_{2}\right)\right] \\
& +\frac{a_{2}}{2 \mu}\left[\delta_{m n} M_{, s l}\left(\mathbf{x}, l_{2}\right)+\delta_{l n} M_{, s m}\left(\mathbf{x}, l_{2}\right)\right]+\frac{2 a_{3}}{\mu} \delta_{m l} M_{, s n}\left(\mathbf{x}, l_{2}\right)-\frac{2 a_{4}}{\mu} \delta_{s n} M_{, m l}\left(\mathbf{x}, l_{2}\right) \\
& -\frac{a_{5}}{\mu} \delta_{s l} M_{, m n}\left(\mathbf{x}, l_{2}\right)-\frac{a_{5}}{\mu} \delta_{s m} M_{, l n}\left(\mathbf{x}, l_{2}\right)-\frac{a_{2}}{2 \mu l_{2}^{2}}\left[\delta_{m n} \delta_{s l}+\delta_{l n} \delta_{s m}\right] M\left(\mathbf{x}, l_{2}\right)-\frac{2 a_{3}}{\mu l_{2}^{2}} \delta_{s n} \delta_{m l} M\left(\mathbf{x}, l_{2}\right)
\end{aligned}
$$

The four tensors $S_{s t m n}, \hat{S}_{s t l m n}, N_{t s m n}, \hat{N}_{r t s l m n}$ are Eshelby tensors of an isotropic second gradient medium, respectively. $S_{s t m n}$ is Eshelby tensor corresponding to the classical one of Cauchy medium. $\hat{S}_{s t l m n}, N_{t s m n}, \hat{N}_{r t s l m n}$ are additional Eshelby tensors due to second gradient theory.

It is seen that Eshelby tensors depends on the following three integrals and their derivatives:

$$
\psi(\mathbf{x})=\frac{1}{4 \pi} \int_{\Omega} r d \mathbf{x}^{\prime}, \quad \phi(\mathbf{x})=\frac{1}{4 \pi} \int_{\Omega} \frac{1}{r} d \mathbf{x}^{\prime}, \quad M(\mathbf{x}, k)=\frac{1}{4 \pi} \int_{\Omega} \frac{e^{-r / k}}{r} d \mathbf{x}^{\prime}
$$

In classical Eshelby tensor, there are only the first and second integrals (Mura, 1987). For inclusion of special shape, such as spherical, cylindrical and ellipsoidal, the two integrals can be derived analytically and the details can be found in Mura (1987). For convenience, these analytical expressions are listed in Appendix B.

The evaluation of the third integral is difficult. But for spherical and cylindrical inclusions their analytical expressions have been given by Cheng and He (1995, 1997). For ellipsoidal inclusion, Ma and Hu (2006) provided a simplified form involving only one-dimensional integral. The detailed expressions of the third integral and its derivatives are listed in Appendix B for spherical, cylindrical and ellipsoidal inclusions respectively.

Here for simplicity, we give only expression of tensor $S_{s t m n}$, which is also the most interested one. It is written as:

$$
S_{s t m n}=S_{\text {stmn }}^{c}+S_{s t m n}^{1}+S_{s t m n}^{2}
$$

where

$$
\begin{aligned}
S_{s t m n}^{c} & =\frac{\lambda+\mu}{\lambda+2 \mu} \psi_{, s t m n}(\mathbf{x})-\frac{\lambda}{\lambda+2 \mu} \delta_{m n} \phi_{, s t}(\mathbf{x})-\frac{1}{2}\left[\delta_{s n} \phi_{, m t}(\mathbf{x})+\delta_{t n} \phi_{, s m}(\mathbf{x})+\delta_{m s} \phi_{, n t}(\mathbf{x})+\delta_{m t} \phi_{, s n}(\mathbf{x})\right] \\
S_{s t m n}^{1} & =\frac{\lambda}{\lambda+2 \mu} \delta_{m n} M_{, s t}\left(\mathbf{x}, l_{1}\right)-\frac{2 \mu l_{1}^{2}}{\lambda+2 \mu}\left[\phi_{, s t m n}(\mathbf{x})-M_{, s t m n}\left(\mathbf{x}, l_{1}\right)\right] \\
S_{s t m n}^{2} & =2 l_{2}^{2}\left[\phi_{, s t m n}(\mathbf{x})-M_{, s t m n}\left(\mathbf{x}, l_{2}\right)\right]+\frac{1}{2}\left[\delta_{s n} M_{, t m}\left(\mathbf{x}, l_{2}\right)+\delta_{t n} M_{, s m}\left(\mathbf{x}, l_{2}\right)+\delta_{m s} M_{, n t}\left(\mathbf{x}, l_{2}\right)+\delta_{m t} M_{, s n}\left(\mathbf{x}, l_{2}\right)\right]
\end{aligned}
$$

where $S_{s t m n}^{c}$ is the classical Eshelby tensor, $S_{\text {stmn }}^{1}$ and $S_{\text {stmn }}^{2}$ are new parts introduced by second gradient theory and depend on the characteristic length $l_{1}$ and $l_{2}$, respectively. When strain gradient effects are neglected, i.e. $l_{1}=l_{2}=0$, the obtained Eshelby tensor Eq. (3.14) is reduced to the classical one $S_{s t m n}^{c}$.

With help of the integrals in Eq. (3.13), analytical expression of $S_{s t m n}$ can be obtained for spherical and cylindrical inclusions. For ellipsoidal inclusion, tensor $S_{\text {stmn }}$ can be derived with only one-dimensional integral in Appendix B.3. The other three Eshelby tensors can also be evaluated in the same way.

\subsection{Average Eshelby tensors}

Unlike Eshelby tensor in Cauchy medium, Eshelby tensors in second gradient medium are not uniform inside the inclusion $\Omega$. In the following, we will give their averages over inclusion domain, which are useful to build homogenization method. 
We calculate volume average over inclusion domain on both sides of Eq. (3.9). It is found that the following properties hold whether for spherical, cylindrical or ellipsoidal inclusions:

$$
\left\langle\hat{S}_{\text {stlmn }}(\mathbf{x})\right\rangle_{I}=0,\left\langle N_{\text {stlmn }}\right\rangle_{I}=0
$$

where $\langle\cdot\rangle_{I}$ means volume average of said quantity over inclusion.

Eq. (3.16) means that in sense of average, Eshelby tensors in second gradient medium are uncoupled. That is:

$$
\left\langle\varepsilon_{s t}(\mathbf{x})\right\rangle_{I}=\left\langle S_{s t m n}(\mathbf{x})\right\rangle_{I} \varepsilon_{m n}^{*},\left\langle\eta_{r t s}(\mathbf{x})\right\rangle_{I}=\left\langle\hat{N}_{r t s l m n}(\mathbf{x})\right\rangle_{I} \eta_{l m n}^{*}
$$

For spherical inclusion, these average Eshelby tensors have analytical expressions and can be written as:

$$
\begin{aligned}
\left\langle S_{s t m n}(\mathbf{x})\right\rangle_{I}= & s_{1} \delta_{s t} \delta_{m n}+s_{2}\left(\delta_{s m} \delta_{t n}+\delta_{s n} \delta_{m t}\right) \\
\left\langle\hat{N}_{r t s l m n}(\mathbf{x})\right\rangle_{I}= & \hat{n}_{1} \delta_{s n} \delta_{m l} \delta_{r t}+\hat{n}_{2}\left(\delta_{n r} \delta_{t s} \delta_{l m}+\delta_{n t} \delta_{r s} \delta_{l m}+\delta_{s l} \delta_{m n} \delta_{r t}+\delta_{s m} \delta_{n l} \delta_{r t}\right) \\
& +\hat{n}_{3}\left(\delta_{s l} \delta_{m r} \delta_{t n}+\delta_{s l} \delta_{m t} \delta_{r n}+\delta_{s m} \delta_{l r} \delta_{t n}+\delta_{s m} \delta_{l t} \delta_{r n}\right)+\hat{n}_{4}\left(\delta_{s n} \delta_{m r} \delta_{l t}+\delta_{s n} \delta_{m t} \delta_{l r}\right) \\
& +\hat{n}_{5}\left(\delta_{m n} \delta_{r s} \delta_{l t}+\delta_{m n} \delta_{t s} \delta_{l r}+\delta_{l n} \delta_{t s} \delta_{m r}+\delta_{l n} \delta_{r s} \delta_{m t}\right)
\end{aligned}
$$

where

$$
\begin{aligned}
& s_{1}=\frac{3 \lambda-2 \mu}{15(\lambda+2 \mu)}+\frac{5 \lambda+2 \mu}{5 R^{3}(\lambda+2 \mu)} \Phi\left(l_{1}\right) \Gamma\left(l_{1}\right)-\frac{2}{5 R^{3}} \Phi\left(l_{2}\right) \Gamma\left(l_{2}\right) \\
& s_{2}=\frac{3 \lambda+8 \mu}{15(\lambda+2 \mu)}+\frac{2 \mu}{5 R^{3}(\lambda+2 \mu)} \Phi\left(l_{1}\right) \Gamma\left(l_{1}\right)+\frac{3}{5 R^{3}} \Phi\left(l_{2}\right) \Gamma\left(l_{2}\right) \\
& \hat{n}_{1}=\frac{\Phi\left(l_{2}\right) \Gamma\left(l_{2}\right)}{35 \mu R^{3} l_{2}^{2}} 2\left(a_{5}-6 a_{4}-28 a_{3}\right)-\frac{\Phi\left(l_{1}\right) \Gamma\left(l_{1}\right)}{35(\lambda+2 \mu) R^{3} l_{1}^{2}}\left[14 a_{3}+7 a_{2}+2\left(a_{4}+a_{5}\right)\right] \\
& \hat{n}_{2}=\frac{\Phi\left(l_{2}\right) \Gamma\left(l_{2}\right)}{35 \mu R^{3} l_{2}^{2}}\left(8 a_{4}-13 a_{5}-28 a_{2}+28 a_{3}\right)-\frac{\Phi\left(l_{1}\right) \Gamma\left(l_{1}\right)}{35(\lambda+2 \mu) R^{3} l_{1}^{2}}\left[8\left(a_{4}+a_{5}\right)+7\left(2 a_{1}+3 a_{2}+4 a_{3}\right)\right] \\
& \hat{n}_{3}=\frac{\Phi\left(l_{2}\right) \Gamma\left(l_{2}\right)}{35 \mu R^{3} l_{2}^{2}}\left(8 a_{4}-38 a_{5}\right)-\frac{\Phi\left(l_{1}\right) \Gamma\left(l_{1}\right)}{35(\lambda+2 \mu) R^{3} l_{1}^{2}} 8\left(a_{4}+a_{5}\right) \\
& \hat{n}_{4}=\frac{\Phi\left(l_{2}\right) \Gamma\left(l_{2}\right)}{35 \mu R^{3} l_{2}^{2}} 4\left(a_{5}-6 a_{4}\right)-\frac{\Phi\left(l_{1}\right) \Gamma\left(l_{1}\right)}{35(\lambda+2 \mu) R^{3} l_{1}^{2}} 4\left(a_{4}+a_{5}\right) \\
& \hat{n}_{5}=\frac{\Phi\left(l_{2}\right) \Gamma\left(l_{2}\right)}{35 \mu R^{3} l_{2}^{2}} 2\left(7 a_{2}+4 a_{4}+4 a_{5}\right)-\frac{\Phi\left(l_{1}\right) \Gamma\left(l_{1}\right)}{35(\lambda+2 \mu) R^{3} l_{1}^{2}}\left[8\left(a_{4}+a_{5}\right)+14\left(2 a_{1}+a_{2}\right)\right]
\end{aligned}
$$

and $\Phi(k)=-k(k+R) e^{-R / k}, \quad \Gamma(k)=R \cosh \left(\frac{R}{k}\right)-k \sinh \left(\frac{R}{k}\right), R$ is radius of spherical inclusion.

For cylindrical inclusion, they are

$$
\begin{aligned}
\left\langle S_{\alpha \beta \gamma \rho}(\mathbf{x})\right\rangle_{I}= & t_{1} \delta_{\alpha \beta} \delta_{\gamma \rho}+t_{2}\left(\delta_{\alpha \gamma} \delta_{\beta \rho}+\delta_{\alpha \rho} \delta_{\beta \gamma}\right) \\
\left\langle\hat{N}_{\alpha \beta \gamma \rho \xi \varsigma}\right\rangle_{I}= & \hat{t}_{1} \delta_{\alpha \beta} \delta_{\gamma \varsigma} \delta_{\xi \rho}+\hat{t}_{2}\left(\delta_{\alpha \varsigma} \delta_{\beta \rho} \delta_{\gamma \xi}+\delta_{\alpha \rho} \delta_{\beta \varsigma} \delta_{\gamma \xi}+\delta_{\gamma \rho} \delta_{\alpha \xi} \delta_{\beta \varsigma}+\delta_{\gamma \rho} \delta_{\alpha \varsigma} \delta_{\beta \xi}\right) \\
& +\hat{t}_{3}\left(\delta_{\alpha \gamma} \delta_{\beta \rho} \delta_{\xi \varsigma}+\delta_{\alpha \rho} \delta_{\beta \gamma} \delta_{\xi \varsigma}+\delta_{\alpha \gamma} \delta_{\beta \xi} \delta_{\rho \varsigma}+\delta_{\alpha \xi} \delta_{\beta \gamma} \delta_{\rho \varsigma}\right)+\hat{t}_{4}\left(\delta_{\alpha \xi} \delta_{\beta \rho} \delta_{\gamma \varsigma}+\delta_{\alpha \rho} \delta_{\beta \xi} \delta_{\gamma \varsigma}\right) \\
& +\hat{t}_{5}\left(\delta_{\alpha \beta} \delta_{\xi \varsigma} \delta_{\gamma \rho}+\delta_{\alpha \beta} \delta_{\rho \varsigma} \delta_{\gamma \xi}+\delta_{\alpha \gamma} \delta_{\beta \varsigma} \delta_{\xi \rho}+\delta_{\alpha \varsigma} \delta_{\beta \gamma} \delta_{\xi \rho}\right)
\end{aligned}
$$

where indices $\alpha, \beta, \gamma, \rho, \xi, \zeta$ range from 1 to 2 , and

$$
\begin{aligned}
& t_{1}=\frac{\lambda-\mu}{4(\lambda+2 \mu)}-\frac{2 \lambda+\mu}{2(\lambda+2 \mu)} \mathrm{I}_{1}\left(\frac{R}{l_{1}}\right) \mathrm{K}_{1}\left(\frac{R}{l_{1}}\right)+\frac{1}{2} \mathrm{I}_{1}\left(\frac{R}{l_{2}}\right) \mathrm{K}_{1}\left(\frac{R}{l_{2}}\right) \\
& t_{2}=\frac{\lambda+3 \mu}{4(\lambda+2 \mu)}-\frac{\mu}{2(\lambda+2 \mu)} \mathrm{I}_{1}\left(\frac{R}{l_{1}}\right) \mathrm{K}_{1}\left(\frac{R}{l_{1}}\right)-\frac{1}{2} \mathrm{I}_{1}\left(\frac{R}{l_{2}}\right) \mathrm{K}_{1}\left(\frac{R}{l_{2}}\right) \\
& \hat{t}_{1}=\frac{18 a_{3}+5 a_{4}-a_{5}}{12 \mu l_{2}{ }^{2}} \mathrm{~K}_{1}\left(\frac{R}{l_{2}}\right) \mathrm{I}_{1}\left(\frac{R}{l_{2}}\right)+\frac{3\left(2 a_{3}+a_{2}\right)+\left(a_{4}+a_{5}\right)}{12 l_{1}{ }^{2}(\lambda+2 \mu)} \mathrm{K}_{1}\left(\frac{R}{l_{1}}\right) \mathrm{I}_{1}\left(\frac{R}{l_{1}}\right) \\
& \hat{t}_{2}=\frac{\left(a_{4}+a_{5}\right)}{12 l_{1}^{2}(\lambda+2 \mu)} \mathrm{K}_{1}\left(\frac{R}{l_{1}}\right) \mathrm{I}_{1}\left(\frac{R}{l_{1}}\right)+\frac{2 a_{5}-a_{4}}{12 \mu l_{2}{ }^{2}} \mathrm{~K}_{1}\left(\frac{R}{l_{2}}\right) \mathrm{I}_{1}\left(\frac{R}{l_{2}}\right) \\
& \hat{t}_{3}=\frac{3\left(2 a_{1}+a_{2}\right)+2\left(a_{4}+a_{5}\right)}{24 l_{1}{ }^{2}(\lambda+2 \mu)} \mathrm{K}_{1}\left(\frac{R}{l_{1}}\right) \mathrm{I}_{1}\left(\frac{R}{l_{1}}\right)-\frac{3 a_{2}-2\left(a_{4}+a_{5}\right)}{24 \mu l_{2}{ }^{2}} \mathrm{~K}_{1}\left(\frac{R}{l_{2}}\right) \mathrm{I}_{1}\left(\frac{R}{l_{2}}\right)
\end{aligned}
$$




$$
\begin{aligned}
& \hat{t}_{4}=\frac{5 a_{4}-a_{5}}{12 \mu l_{2}{ }^{2}} \mathrm{~K}_{1}\left(\frac{R}{l_{2}}\right) \mathrm{I}_{1}\left(\frac{R}{l_{2}}\right)+\frac{\left(a_{4}+a_{5}\right)}{12 l_{1}{ }^{2}(\lambda+2 \mu)} \mathrm{K}_{1}\left(\frac{R}{l_{1}}\right) \mathrm{I}_{1}\left(\frac{R}{l_{1}}\right) \\
& \hat{t}_{5}=\frac{9 a_{2}-6 a_{3}-4 a_{4}+2 a_{5}}{24 \mu l_{2}^{2}} \mathrm{~K}_{1}\left(\frac{R}{l_{2}}\right) \mathrm{I}_{1}\left(\frac{R}{l_{2}}\right)+\frac{3\left(2 a_{1}+3 a_{2}+4 a_{3}\right)+4\left(a_{4}+a_{5}\right)}{24 l_{1}{ }^{2}(\lambda+2 \mu)} \mathrm{K}_{1}\left(\frac{R}{l_{1}}\right) \mathrm{I}_{1}\left(\frac{R}{l_{1}}\right)
\end{aligned}
$$

$\mathrm{I}_{1}\left(\frac{z}{k}\right)$ and $\mathrm{K}_{1}\left(\frac{R}{k}\right)$ are the first order modified Bessel functions of the first kind and second kind respectively. $R$ is radius of cylindrical inclusion.

For ellipsoidal inclusion, full analytical expressions of these average Eshelby tensors are difficult to be derived. But with the help of formulas in Appendix B.3, these average Eshelby tensors over an ellipsoidal inclusion can be computed easily.

\section{Connection with existing simplified strain gradient theories}

As discussed in introduction, some well-known simplified strain gradient theories, such as couple stress theory, Aifantis and Kleinert and Wei \& Hutchinson's models, have similar forms of governing equation (Gao \& Ma, 2009; Zhang \& Sharma, 2005; Zheng \& Zhao, 2004) as that of the general isotropic second gradient theory, it is therefore interesting to explore their connection. In the following, we focus on Eshelby tensor $S_{s t m n}$ for simplicity, and the other Eshelby tensors can be analyzed in the same way.

\subsection{Couple stress theory (abbreviated as CS)}

Couple stress theory is the form III in Mindlin (1964) theory with only rotation gradient for micro-deformation gradient. According to its strain energy density function (Zheng \& Zhao, 2004), by setting

$$
a_{1}=0, \quad a_{2}=0, \quad a_{3}=0, \quad a_{4}=\frac{1}{2} \mu l_{c s}^{2}, \quad a_{5}=-\frac{1}{2} \mu l_{c s}^{2}
$$

connection of the two characteristic lengths in second gradient theory and CS are obtained:

$$
l_{1}=0, \quad l_{2}=l_{c s}
$$

where $l_{c S}$ is material constant with dimension of length in CS theory. Eq. (4.2) shows that there is only one characteristic length $l_{2}$ in CS.

Substituting Eq. (4.2) to Eq. (2.7), Eq. (2.17) and Eq. (3.14), we obtain the same governing equation and Green's function of couple stress theory. Eshelby tensors for spherical, cylindrical and ellipsoidal inclusions of couple stress theory can also be obtained by using Eqs. (4.1) and (4.2), the results of Zheng and Zhao (2004) for spherical inclusion is found as a special case.

\subsection{Aifantis strain gradient theory (abbreviated as ASG)}

ASG theory is the form II in Mindlin (1964) theory with special definition of high-order stress. According to its strain energy density function (Gao \& Ma, 2009), by setting

$$
a_{1}=\frac{1}{2} \lambda l_{\text {Aifantis }}^{2}, \quad a_{2}=0, \quad a_{3}=0, \quad a_{4}=\frac{1}{2} \mu l_{\text {Aifantis }}^{2}, \quad a_{5}=\frac{1}{2} \mu l_{\text {Aifantis }}^{2}
$$

we obtain also the following relation between the characteristic lengths of ASG and second gradient theory:

$$
l_{1}=l_{2}=l_{\text {Aifantis }}
$$

where $l_{\text {Aifantis }}$ is material length scale parameter. It is seen that ASG theory has two equal characteristic lengths.

Using Eqs. (4.3) and (4.4), the governing equation, Green's function and Eshelby tensors of ASG are obtained, which are consistent with those in Gao and Ma (2009).

\subsection{Kleinert strain gradient theory (KSG)}

According to the strain energy density function of KSG (Zhang \& Sharma, 2005), we set

$$
a_{1}=\frac{\lambda+2 \mu}{2} l_{\text {Kleinert }}^{2}-\frac{1}{2} \mu l_{\text {Kleinert }}^{2}, \quad a_{3}=\frac{1}{2} \mu l_{\text {Kleinert }}^{2}, a_{2}=a_{4}=a_{5}=0
$$

then we have:

$$
l_{1}=l_{\text {Kleinert }}^{\prime}, \quad l_{2}=l_{\text {Kleinert }}
$$

It is seen that the characteristic lengths in second gradient theory are the corresponding characteristic lengths in KSG.

Similarly, the governing equation, Green's function and Eshelby tensor of KSG can be obtained directly by using Eqs. (4.5) and (4.6) in our work. 


\subsection{Wei-Hutchinson strain gradient theory (W-HSG)}

Inclusion problem of W-HSG has not been examined yet. Here we will derive it as a special case of our results.

According to the strain energy density function of W-HSG, we set

$$
a_{1}=0, \quad a_{2}=0, \quad a_{3}=0, \quad a_{4}=E l_{W-H}^{2}, \quad a_{5}=0
$$

Then two corresponding characteristic lengths are respectively:

$$
l_{1}=\sqrt{\frac{2 E}{\lambda+2 \mu}} l_{W-H}=h_{1} l_{W-H}, \quad l_{2}=\sqrt{\frac{2 E}{\mu}} l_{W-H}=h_{2} l_{W-H}
$$

where $l_{W-H}$ is material length parameter in W-HSG, $E$ is Young's modulus. It shows that W-HSG can be considered as special case of second gradient theory specifically with Eqs. (4.7) and (4.8).

Substituting Eqs. (4.7) and (4.8) to Eqs. (2.7), (2.17) and (3.14), we obtain the displacement balance equation:

$$
(\lambda+2 \mu) \nabla \nabla \cdot \mathbf{u}-\mu \nabla \times \nabla \times \mathbf{u}-2 E l_{W-H}^{2} \nabla^{4} \mathbf{u}+\mathbf{f}=0
$$

or in vector component presentation:

$$
(\lambda+\mu) u_{j, j i}+\mu u_{i, k k}-2 E l_{W-H}^{2}\left(u_{i, k k}\right)_{, l l}=0
$$

Green's function is given by:

$$
\begin{aligned}
G_{i j}= & G_{i j}^{c}+G_{i j}^{1}+G_{i j}^{2} \\
= & \frac{1}{8 \pi \mu}\left(\frac{2}{r} \delta_{i j}-\frac{\lambda+\mu}{\lambda+2 \mu} r_{, i j}\right)+\frac{h_{1}^{2} l_{W-H}^{2}}{4 \pi(\lambda+2 \mu)}\left(\frac{1-e^{-r /\left(h_{1} l_{W-H}\right)}}{r}\right)_{, i j} \\
& -\frac{h_{2}^{2} l_{W-H}^{2}}{4 \pi \mu}\left(\frac{1-e^{-r /\left(h_{2} l_{W-H}\right)}}{r}\right)_{, i j}-\frac{1}{4 \pi \mu} \frac{e^{-r /\left(h_{2} l_{W-H}\right)}}{r} \delta_{i j}
\end{aligned}
$$

and Eshelby tensors of W-HSG are:

$$
\begin{aligned}
S_{s t m n}= & S_{s t m n}^{c}+S_{s t m n}^{1}+S_{s t m n}^{2} \\
S_{s t m n}^{c}= & \frac{\lambda+\mu}{\lambda+2 \mu} \psi_{, s t m n}(\mathbf{x})-\frac{\lambda}{\lambda+2 \mu} \delta_{m n} \phi_{, s t}(\mathbf{x})-\frac{1}{2}\left[\delta_{s n} \phi_{, m t}(\mathbf{x})+\delta_{t n} \phi_{, s m}(\mathbf{x})+\delta_{m s} \phi_{, n t}(\mathbf{x})+\delta_{m t} \phi_{, s n}(\mathbf{x})\right] \\
S_{s t m n}^{1}= & \frac{\lambda}{\lambda+2 \mu} \delta_{m n} M_{, s t}\left(\mathbf{x}, h_{1} l_{W-H}\right)-\frac{2 \mu h_{1}^{2} l_{W-H}^{2}}{\lambda+2 \mu}\left[\phi_{, s t m n}(\mathbf{x})-M_{, s t m n}\left(\mathbf{x}, h_{1} l_{W-H}\right)\right] \\
S_{s t m n}^{2}= & 2 h_{2}^{2} l_{W-H}^{2}\left[\phi_{, s t m n}(\mathbf{x})-M_{, s t m n}\left(\mathbf{x}, h_{2} l_{W-H}\right)\right] \\
& +\frac{1}{2}\left[\delta_{s n} M_{, t m}\left(\mathbf{x}, h_{2} l_{W-H}\right)+\delta_{t n} M_{s m}\left(\mathbf{x}, h_{2} l_{W-H}\right)+\delta_{m s} M_{, n t}\left(\mathbf{x}, h_{2} l_{W-H}\right)+\delta_{m t} M_{, s n}\left(\mathbf{x}, h_{2} l_{W-H}\right)\right]
\end{aligned}
$$

It is proved that these results can also be obtained directly by the method in Section 2 and 3.

\section{Numerical examples}

In this section, some numerical examples will be given to illustrate property of Eshelby tensor of second gradient theory. And Eshelby tensors of special strain gradient theories discussed in Section 4 will also be included. For simplicity, only spherical inclusion is considered in the following.

For spherical inclusion in second gradient medium, analytical expression of Eshelby tensor can be obtained with help of Eq. (3.13) (detail see Appendix B.1) and Eq. (3.14). Only $S_{1111}$ and $S_{1122}$ are computed in the following. $R$ is radius of the spherical inclusion. Lame constants used in computation are $\lambda=50 \mathrm{GPa}, \mu=26 \mathrm{GPa}$. We set $l_{2}=1 \mu \mathrm{m}$, and $l_{1}$ is proportional to $l_{2}$. Therefore, for CS there are $l_{1}=0, l_{2}=l_{c S}=1 \mu \mathrm{m}$, for ASG there are $l_{1}=l_{2}=l_{\text {Aifantis }}=1 \mu \mathrm{m}$, and for W-HSG, there are $l_{1}=0.5 l_{2}=0.5 \mu \mathrm{m}$. The two characteristic lengths of KSG equal to $l_{1}$ and $l_{2}$ respectively so that the result of KSG can be considered as the same as those of second gradient theory, but they also set $a_{2}=a_{4}=a_{5}=0$.

Fig. 1 shows variation of components $S_{1111}$ and $S_{1122}$ along axis $x_{1}$ with different values of $l_{1}$. The radius of inclusion is $R=1 l_{2}$. The corresponding results of CS, ASG, W-HSG and the classical model (calculated by Eq. (3.15a)) are also included. It can be seen that all Eshelby tensors of strain gradient models vary with position inside of spherical inclusion. As shown in Fig. $1 \mathrm{a}, S_{1111}$ decreases with increase of $x_{1}$ for all second gradient models. The classical Cauchy model predicts the largest value of $S_{1111}$, and different models control amplitude and variation of $S_{1111}$. From Fig. $1 \mathrm{~b}$, it can be seen that, the length parameter $l_{1}$ has a significant influence on $S_{1122}$, i.e. different strain gradient models have quite different values of $S_{1122}$. With increase of $x_{1}, S_{1122}$ increases slightly in CS, decreases in W-HSG and ASG. The value of $S_{1122}$ is larger than that in classical medium for CS medium and smaller for ASG medium. 


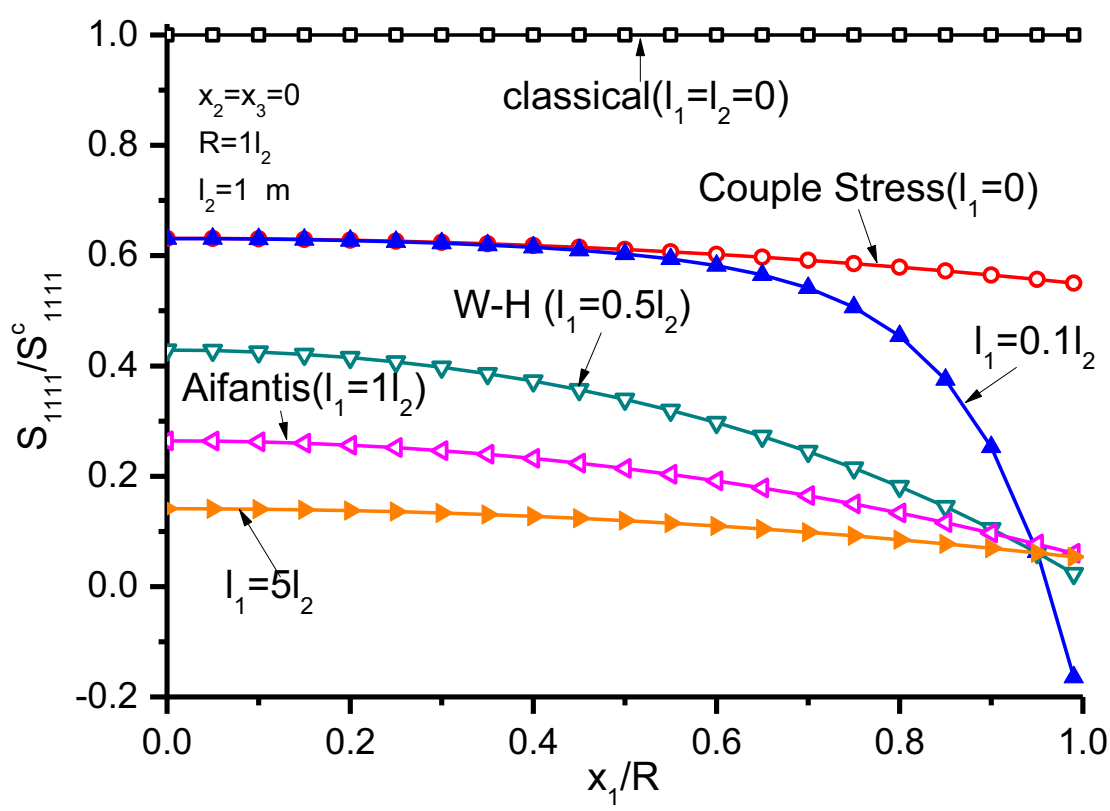

(a) $S_{1111}$

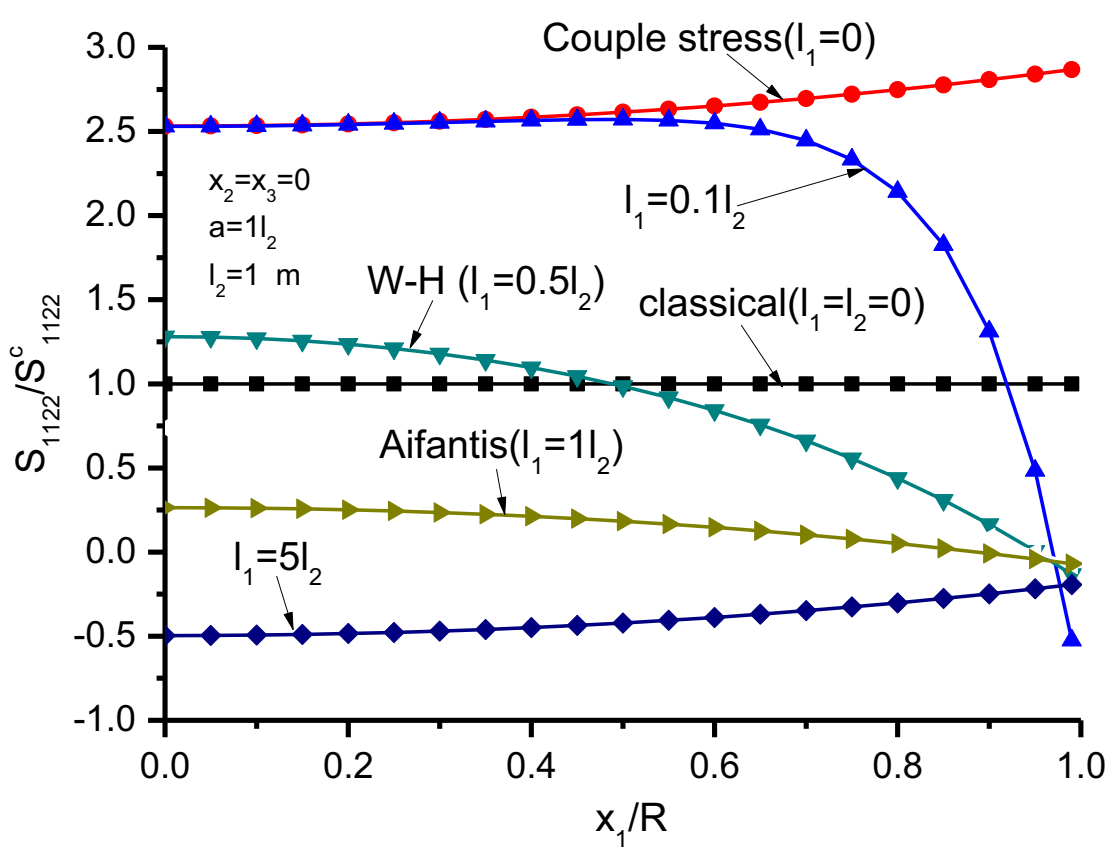

(b) $S_{1122}$

Fig. 1. Variation of Eshelby tensor component along axis $x_{1}$ with different $l_{1}$. (a) $S_{1111}$, (b) $S_{1122}$. 


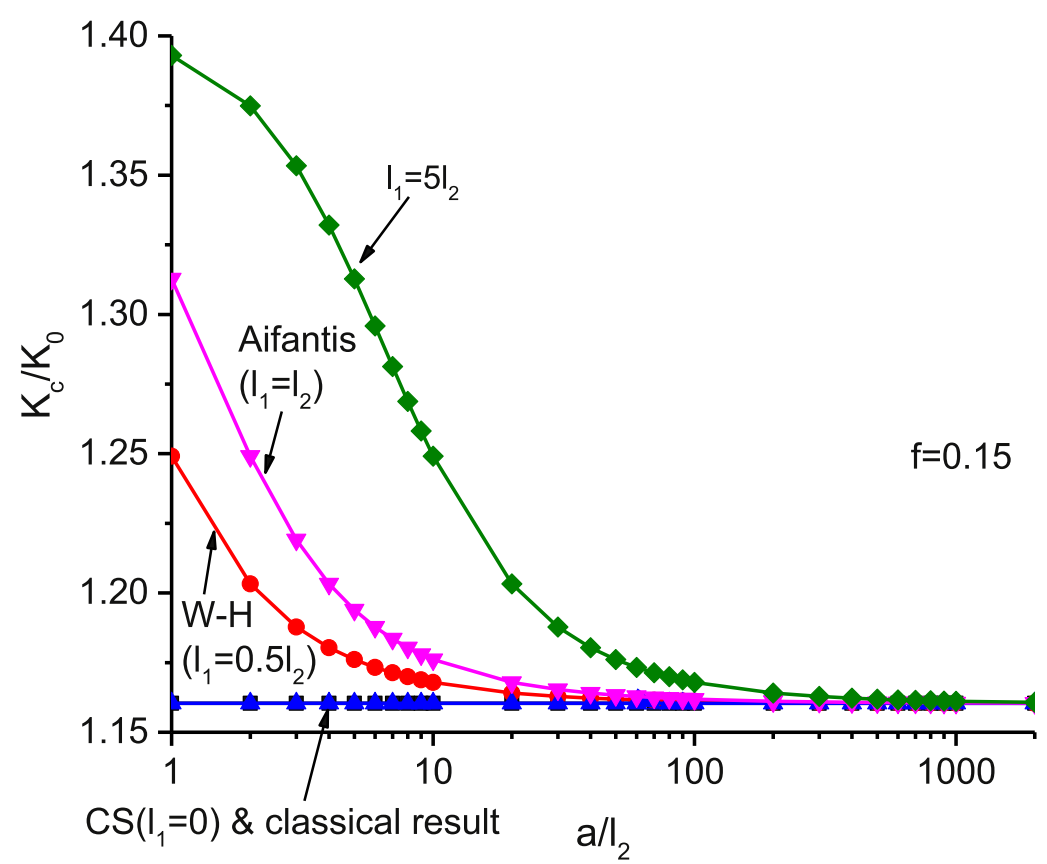

(a) $K_{c}$

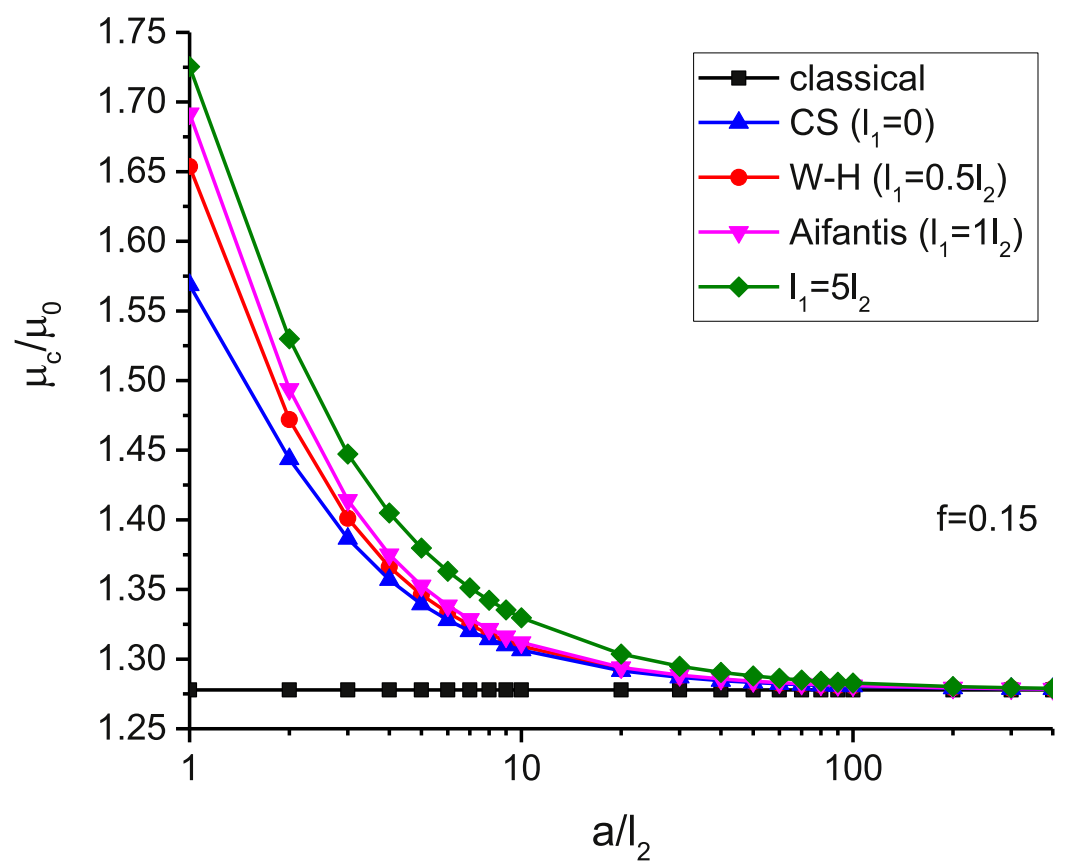

(b) $\mu_{c}$

Fig. 2. Variations of the effective moduli as a function of the size of inclusion. (a) $K_{c}$ (b) $\mu_{c}$. 


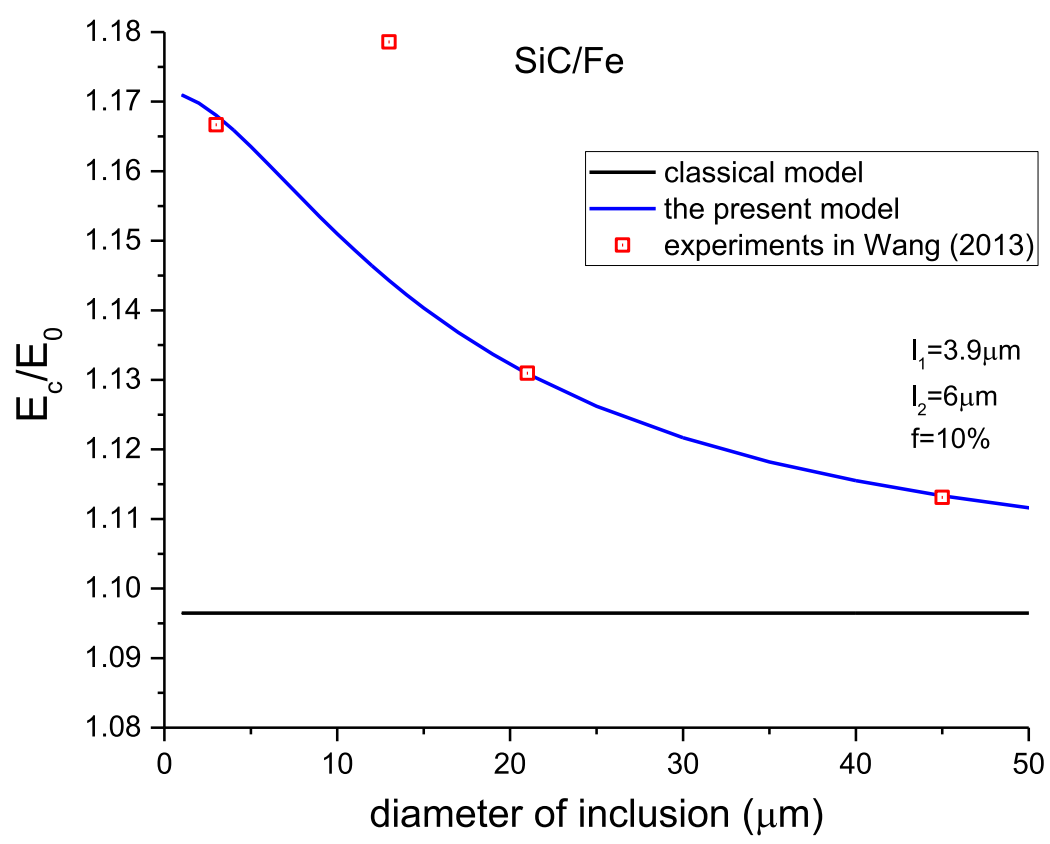

(a) $\mathrm{SiC} / \mathrm{Fe}$

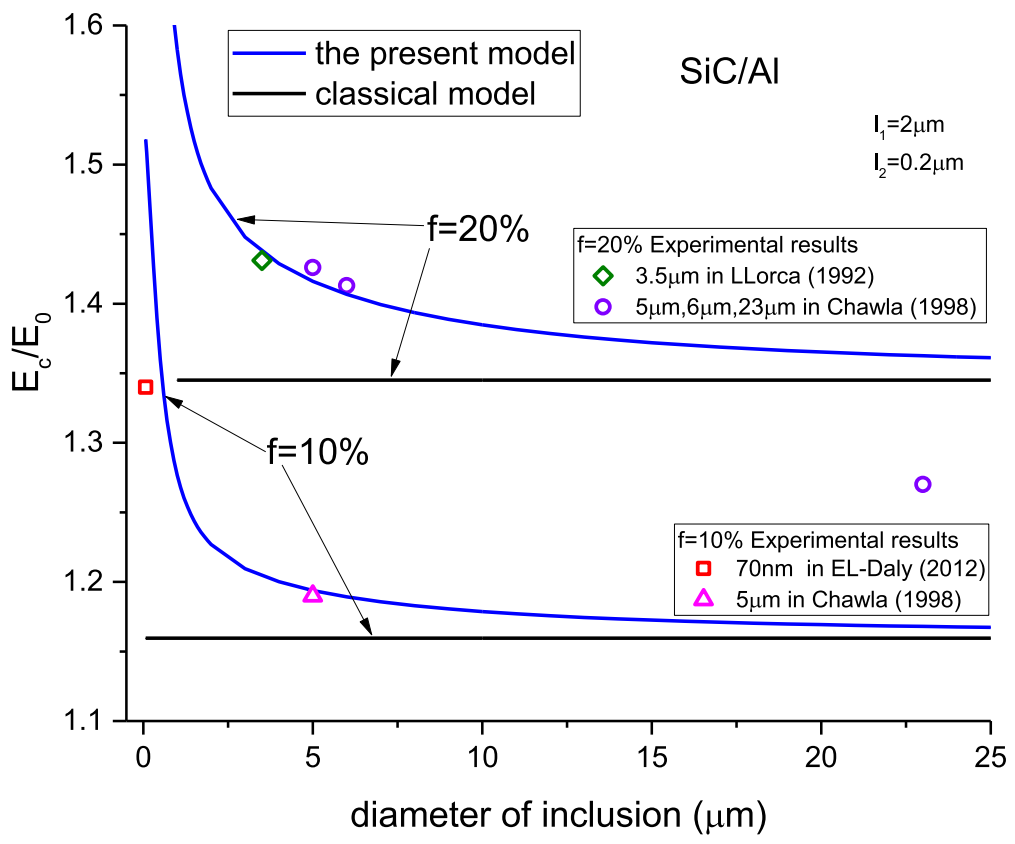

(b) $\mathrm{SiC} / \mathrm{Al}$

Fig. 3. Comparison between the present model and different experimental results for the effective Young's modulus. (a) Effective Young's modulus of $10 \% \mathrm{SiC} / \mathrm{Fe}$ vs. inclusion size (b) Effective Young's modulus of $10 \%$ and $20 \% \mathrm{SiC} / \mathrm{Al}$ vs. inclusion size. 


\section{Effective modulus}

For isotropic composite composed of a matrix of second gradient medium and spherical inclusions, according to the average equivalent inclusion method and the generalized Mori-Tanaka method (Liu \& Hu, 2005), effective bulk modulus $K_{c}$ and effective shear modulus $\mu_{c}$ can be obtained analytically:

$$
\begin{aligned}
& K_{c}=K_{0}\left[1+\frac{f}{(1-f) S_{\alpha}+\frac{K_{0}}{K_{1}-K_{0}}}\right] \\
& \mu_{c}=\mu_{0}\left[1+\frac{f}{(1-f) S_{\beta}+\frac{\mu_{0}}{\mu_{1}-\mu_{0}}}\right]
\end{aligned}
$$

where

$$
\begin{aligned}
& S_{\alpha}=3 s_{1}+2 s_{2}=\frac{3 K_{0}}{3 K_{0}+4 \mu_{0}}+\frac{9 K_{0}}{a^{3}\left(3 K_{0}+4 \mu_{0}\right)} \Phi\left(l_{1}\right) \Gamma\left(l_{1}\right) \\
& S_{\beta}=2 s_{2}=\frac{6\left(K_{0}+2 \mu_{0}\right)}{5\left(3 K_{0}+4 \mu_{0}\right)}+\frac{12 \mu_{0}}{5 a^{3}\left(3 K_{0}+4 \mu_{0}\right)} \Phi\left(l_{1}\right) \Gamma\left(l_{1}\right)+\frac{6}{5 a^{3}} \Phi\left(l_{2}\right) \Gamma\left(l_{2}\right)
\end{aligned}
$$

$K_{0}, \mu_{0}, K_{1}, \mu_{1}$ are bulk modulus and shear modulus of the matrix and inclusion respectively, $l_{1}, l_{2}$ are two characteristic lengths of the matrix, $f$ is volume fraction of inclusion, $s_{1}, s_{2}$ are defined in Eq. (3.19).

Variations of $K_{c}$ and $\mu_{c}$ as function of inclusion size with different $l_{1}$ are shown in Fig. 2a and b respectively. The material parameters are $K_{1}=247 \mathrm{GPa}, \mu_{1}=209 \mathrm{GPa}, K_{0}=67 \mathrm{GPa}, \mu_{1}=26 \mathrm{GPa}$ and $f=0.15$. Both $K_{c}$ and $\mu_{c}$ decrease with increase of inclusion size, and tend to classical result for large size of inclusion for all models. $K_{c}$ with CS model is the same as classical result due to only rotation gradient considered, as expected.

We have compared the effective Young's modulus predicted by the present model with several experimental results in literatures, and the classical results are also included. Composites with Fe matrix and 10\% SiC particles with different sizes of 3, 13, 21, $45 \mu \mathrm{m}$ are taken for comparison, which was presented by Wang, Zhang, Zong and Yang (2013). Eq. (6.1) and $E_{c}=\frac{9 K_{c} \mu_{c}}{3 K_{c}+\mu_{c}}$ are used to evaluate the effective Young's modulus. The characteristic lengths of strain gradient matrix are set to be $l_{1}=3.9 \mu \mathrm{m}, l_{2}=6 \mu \mathrm{m}$. And other material constants are taken form the experiment, which are $E_{1}=450 \mathrm{GPa}, v_{1}=0.17$ for $\mathrm{SiC}$ and $E_{0}=168 \mathrm{GPa}, v_{0}=0.25$ for Fe matrix. As shown in Fig. 3(a), our results agree with the experiments while the classical method cannot characterize the size-dependence of particles. It should be mentioned that this size-dependence is not significant in elasticity unless the size of particle reaches nanoscale, the interface effect becomes pronounced, and however it will become much more significant for overall plastic property of composites.

The comparison of effective Young's modulus between classical results, the present model and experimental results are shown in Fig. 3(b) for SiC/Al composites with different inclusion sizes and different inclusion volume fractions. In Fig 3(b), hollow symbols are the experimental results taken from Chawla, Jones, Andres, and Allison (1998) for 2080Al with different SiC sizes of 5, 6, $23 \mu \mathrm{m}$, El-Daly, Abdelhameed, Hashish, and Eid (2012) for Al with $70 \mathrm{~nm}$ SiC particles and Llorca, Suresh, and Needleman (1992) for 2124Al with $3.5 \mu \mathrm{m} \mathrm{SiC} \mathrm{particles.} \mathrm{The} \mathrm{blue} \mathrm{and} \mathrm{black} \mathrm{lines} \mathrm{are} \mathrm{estimated} \mathrm{by} \mathrm{our} \mathrm{model} \mathrm{and}$ classical model respectively. The material constants used in calculations are $E_{1}=450 \mathrm{GPa}, v_{1}=0.17$ for $\mathrm{SiC}$ and $E_{0}=75 \mathrm{GPa}$ (Chawla et al., 1998), $v_{0}=0.33$ for Al matrix and the two characteristic lengths of strain gradient matrix are $l_{1}=2 \mu$ m, $l_{2}=0.2 \mu \mathrm{m}$. Again the proposed model can capture the dependence of overall elastic property on particle size.

Our elastic micromechanical model predict successfully the size-dependence of Young's and it can be easily extended to predicted elastoplastic behavior (Liu \& Hu, 2005), where size effect is much more pronounced.

\section{Conclusions}

Inclusion problem of linear isotropic second gradient continuum is examined in this paper. Green's function is firstly derived in analytical form, and Eshelby tensors are obtained for inclusion of arbitrary shape. They are all composed of three parts, one is of classical part, and the other two are related to the characteristic lengths of second gradient theory. Analytical Eshelby tensors and their averages are obtained for spherical, cylindrical and ellipsoidal inclusion. It is found that Eshelby tensors are not uniform inside of inclusion, their variations depend intimately on the two characteristic lengths of second gradient theory. We also demonstrate that Green's functions and Eshelby tensors of some special second gradient theories, including couple stress, Aifantis, Kleinert and Wei-Hutchinson strain gradient models, are special cases of our results by proper definition of $l_{1}, l_{2}$. Finally, effective modulus of isotropic composite with spherical inclusions and matrix of second gradient medium are provided analytically. It is shown that influence of inclusion's size can be taken into account and classical results are recovered when size of inclusion is large enough. Our results have fundamental importance in building different homogenization methods to predict overall property of composites. 


\section{Acknowledgements}

This work is supported by National Natural Science Foundation of China under Grants no. 11572329, 11432014, 11672301, 11372318, 11672296, 11521202 and by the Strategic Priority Research Program of the Chinese Academy of Sciences, Grant no. XDB22040501.

\section{Appendices}

A. The solution of Eq. (2.7)

Following Sandru (1966) and the defined differential operators Eq. (2.9), the solution of Eq. (2.7) is:

$$
\mathbf{u}=\square_{1} \mathbf{F}-\square_{2} \nabla \nabla \cdot \mathbf{F}
$$

where : $\square_{1} \square_{3} \mathbf{F}=-\mathbf{f}$

body force $\mathbf{f}$ can be decomposed into:

$$
\mathbf{f}=\nabla \Pi_{0}+\nabla \times \boldsymbol{\Pi}
$$

Supposing the body force $\mathbf{f}$ is a rotational-free field, we have

$$
\mathbf{f}=\nabla \Pi_{0}
$$

If we define

$$
\square_{3} \mathbf{F}=\nabla \Lambda_{0}
$$

then Eq. (A.2) can be rewritten as:

$$
\square_{1} \Lambda_{0}=-\Pi_{0}
$$

with help of $\nabla \nabla \cdot \mathbf{F}=\nabla \times \nabla \times \mathbf{F}+\Delta \mathbf{F}$ and $\square_{1}=\square_{2} \Delta+\square_{3}$, Eq. (A.1) is

$$
\mathbf{u}=\nabla \Lambda_{0}
$$

Now we suppose the body force $\mathbf{f}$ is a solenoidal field, we have

$$
\mathbf{f}=\nabla \times \boldsymbol{\Pi}
$$

and if : $\square_{1} \mathbf{F}=\nabla \times \Lambda$

Eq. (A.2) can be rewritten as:

$$
\square_{3} \Lambda=-\Pi
$$

then the Eq. (A.1) is

$$
\mathbf{u}=\nabla \times \boldsymbol{\Lambda}
$$

Therefore, $\mathbf{u}=\nabla \Lambda_{0}+\nabla \times \Lambda$ is solution of Eq. (2.7).

B. Expressions of three integrals in Eq. (3.13)

B.1. Spherical inclusion

$$
\begin{aligned}
& \psi(\mathbf{x})=-\frac{1}{60}\left(r^{4}-10 R^{2} r^{2}-15 R^{4}\right) \\
& \text { and } \psi_{, i j k l}=-\frac{2}{15}\left(\delta_{i j} \delta_{k l}+\delta_{i k} \delta_{j l}+\delta_{j k} \delta_{i l}\right) \\
& \phi(\mathbf{x})=-\frac{1}{6}\left(r^{2}-3 R^{2}\right) \\
& \text { and } \phi_{, i j}=-\frac{1}{3} \delta_{i j}
\end{aligned}
$$


The analytical expressions of the third integral for a spherical inclusion are given by Cheng and He (1995):

$$
M(\mathbf{x}, k)=k^{2}+\Phi(k) k \frac{\sinh (r / k)}{r}
$$

where: $\Phi(k)=-k(k+R) e^{-R / k}, r=\sqrt{x_{1}^{2}+x_{2}^{2}+x_{3}^{2}}, R$ is the radius of the spherical inclusion.

To obtain its derivatives, $N(r, k)=M(\mathbf{x}, k)$ and $D=\frac{1}{r} \frac{d}{d r}$ are defined, then,

$$
\begin{aligned}
& \frac{\partial M}{\partial x_{i}}=\frac{d N}{d r} \frac{\partial r}{\partial x_{i}}=x_{i} D N \\
& \frac{\partial}{\partial x_{i}}\left(D^{l} N\right)=x_{i} D^{l+1} N \\
& \text { so: } M_{, i}(\mathbf{x}, k)= x_{i} D N \\
& M_{, i j}(\mathbf{x}, k)=\left(\delta_{i j} x_{k}+\delta_{i k} x_{j}+\delta_{j k} x_{i}\right) D^{2} N+x_{i} x_{j} x_{k} D^{3} N \\
& M_{, i j k l}(\mathbf{x}, k)=\tilde{A}_{i j k l} D^{2} N+\tilde{B}_{i j k l} D^{3} N+x_{i} x_{j} x_{k} x_{l} D^{4} N \\
& M_{, i j k l m}(\mathbf{x}, k)= \tilde{C}_{i j k l m} D^{3} N+\tilde{D}_{i j k l m} D^{4} N+x_{i} x_{j} x_{k} x_{l} x_{m} D^{5} N \\
& M_{, i j k l m n}(\mathbf{x}, k)= \tilde{E}_{i j k l m n} D^{3} N+\tilde{F}_{i j k l m n} D^{4} N+\tilde{G}_{i j k l m n} D^{5} N+x_{i} x_{j} x_{k} x_{l} x_{m} x_{n} D^{6} N
\end{aligned}
$$

where:

$$
\begin{aligned}
\Phi(k) & =-k(k+R) e^{-R / k}, \quad \Gamma(r, k)=r \cosh \left(\frac{r}{k}\right)-k \sinh \left(\frac{r}{k}\right) \\
D N & =\frac{\Phi(k)}{r^{3}} \Gamma(r, k) \\
D^{2} N & =\frac{\Phi(k)}{r^{3}}\left[\frac{\sinh \left(\frac{r}{k}\right)}{k}-\frac{3 \Gamma(r, k)}{r^{2}}\right] \\
D^{2} N & =\frac{\Phi(k)}{r^{3}}\left[\frac{\sinh \left(\frac{r}{k}\right)}{k}-\frac{3 \Gamma(r, k)}{r^{2}}\right] \\
D^{4} N & =\frac{\Phi(k)}{r^{5}}\left[-\frac{105 \Gamma(r, k)}{r^{4}}+\frac{45 \sinh \left(\frac{r}{k}\right)}{k r^{2}}-\frac{10 \cosh \left(\frac{r}{k}\right)}{k^{2} r}+\frac{\sinh \left(\frac{r}{k}\right)}{k^{3}}\right] \\
D^{5} N & =\frac{\Phi(k)}{r^{7}}\left[\frac{945 \Gamma(r, k)}{r^{4}}-\frac{420 \sinh \left(\frac{r}{k}\right)}{k r^{2}}+\frac{105 \cosh \left(\frac{r}{k}\right)}{k^{2} r}-\frac{15 \sinh \left(\frac{r}{k}\right)}{k^{3}}+\frac{r \cosh \left(\frac{r}{k}\right)}{k^{4}}\right] \\
D^{6} N & =\frac{\Phi(k)}{r^{7}}\left[-\frac{10395 \Gamma(r, k)}{r^{6}}+\frac{4725 \sinh \left(\frac{r}{k}\right)}{k r^{4}}-\frac{525 \cosh \left(\frac{r}{k}\right)}{k^{2} r^{3}}+\frac{210 \sinh \left(\frac{r}{k}\right)}{k^{3} r^{2}}-\frac{21 \cosh \left(\frac{r}{k}\right)}{k^{4} r}+\frac{\sinh \left(\frac{r}{k}\right)}{k^{5}}\right]
\end{aligned}
$$

and

$$
\begin{aligned}
& \tilde{A}_{i j k l}=\delta_{i j} \delta_{k l}+\delta_{i k} \delta_{j l}+\delta_{j k} \delta_{i l} \\
& \tilde{B}_{i j k l}=\delta_{i j} x_{k} x_{l}+\delta_{i k} x_{j} x_{l}+\delta_{j k} x_{i} x_{l}+\delta_{i l} x_{j} x_{k}+\delta_{j l} x_{i} x_{k}+\delta_{l k} x_{i} x_{j} \\
& \tilde{C}_{i j k l m}=\delta_{i j} \delta_{k l} x_{m}+\delta_{i k} \delta_{j l} x_{m}+\delta_{j k} \delta_{i l} x_{m}+\delta_{i j} \delta_{k m} x_{l}+\delta_{i j} \delta_{l m} x_{k}+\delta_{i k} \delta_{j m} x_{l}+\delta_{i k} \delta_{l m} x_{j}+\delta_{j k} \delta_{i m} x_{l} \\
& +\delta_{j k} \delta_{l m} x_{i}+\delta_{i l} \delta_{j m} x_{k}+\delta_{i l} \delta_{k m} x_{j}+\delta_{j l} \delta_{i m} x_{k}+\delta_{j l} \delta_{k m} x_{i}+\delta_{l k} \delta_{i m} x_{j}+\delta_{l k} \delta_{j m} x_{i} \\
& \tilde{D}_{i j k l m}=\delta_{i j} x_{k} x_{l} x_{m}+\delta_{i k} x_{j} x_{l} x_{m}+\delta_{j k} x_{i} x_{l} x_{m}+\delta_{i l} x_{j} x_{k} x_{m}+\delta_{j l} x_{i} x_{k} x_{m}+\delta_{l k} x_{i} x_{j} x_{m} \\
& +\delta_{i m} x_{j} x_{k} x_{l}+\delta_{j m} x_{i} x_{k} x_{l}+\delta_{k m} x_{i} x_{j} x_{l}+\delta_{l m} x_{i} x_{j} x_{k} \\
& \tilde{E}_{i j k l m n}=\delta_{i j} \delta_{k l} \delta_{m n}+\delta_{i k} \delta_{j l} \delta_{m n}+\delta_{j k} \delta_{i l} \delta_{m n}+\delta_{i j} \delta_{k m} \delta_{l n}+\delta_{i j} \delta_{l m} \delta_{k n}+\delta_{i k} \delta_{j m} \delta_{l n}+\delta_{i k} \delta_{l m} \delta_{j n}+\delta_{j k} \delta_{i m} \delta_{l n} \\
& +\delta_{j k} \delta_{l m} \delta_{i n}+\delta_{i l} \delta_{j m} \delta_{k n}+\delta_{i l} \delta_{k m} \delta_{j n}+\delta_{j l} \delta_{i m} \delta_{k n}+\delta_{j l} \delta_{k m} \delta_{n i}+\delta_{l k} \delta_{i m} \delta_{j n}+\delta_{l k} \delta_{j m} \delta_{n i} \\
& \tilde{F}_{i j k l m n}=\delta_{i j} \delta_{k l} x_{m} x_{n}+\delta_{i k} \delta_{j l} x_{m} x_{n}+\delta_{j k} \delta_{i l} x_{m} x_{n}+\delta_{i j} \delta_{k m} x_{l} x_{n}+\delta_{i j} \delta_{l m} x_{k} x_{n}+\delta_{i k} \delta_{j m} x_{l} x_{n}+\delta_{i k} \delta_{l m} x_{j} x_{n}+\delta_{j k} \delta_{i m} x_{l} x_{n} \\
& +\delta_{j k} \delta_{l m} x_{i} x_{n}+\delta_{i l} \delta_{j m} x_{k} x_{n}+\delta_{i l} \delta_{k m} x_{j} x_{n}+\delta_{j l} \delta_{i m} x_{k} x_{n}+\delta_{j l} \delta_{k m} x_{i} x_{n}+\delta_{l k} \delta_{i m} x_{j} x_{n}+\delta_{l k} \delta_{j m} x_{i} x_{n}+\delta_{i j} \delta_{k n} x_{l} x_{m} \\
& +\delta_{i k} \delta_{j n} x_{l} x_{m}+\delta_{j k} \delta_{i n} x_{l} x_{m}+\delta_{i l} \delta_{j n} x_{k} x_{m}+\delta_{j l} \delta_{i n} x_{k} x_{m}+\delta_{l k} \delta_{i n} x_{j} x_{m}+\delta_{i m} \delta_{j n} x_{k} x_{l}+\delta_{j m} \delta_{i n} x_{k} x_{l}+\delta_{k m} \delta_{n i} x_{j} x_{l} \\
& +\delta_{l m} \delta_{i n} x_{j} x_{k}+\delta_{i j} \delta_{n l} x_{k} x_{m}+\delta_{i k} \delta_{n l} x_{j} x_{m}+\delta_{j k} \delta_{n l} x_{i} x_{m}+\delta_{i l} \delta_{k n} x_{j} x_{m}+\delta_{j l} \delta_{k n} x_{i} x_{m}+\delta_{l k} \delta_{j n} x_{i} x_{m}+\delta_{i m} \delta_{k n} x_{j} x_{l} \\
& +\delta_{j m} \delta_{k n} x_{i} x_{l}+\delta_{k m} \delta_{j n} x_{i} x_{l}+\delta_{l m} \delta_{n j} x_{i} x_{k}+\delta_{i j} \delta_{m n} x_{k} x_{l}+\delta_{i k} \delta_{m n} x_{j} x_{l}+\delta_{j k} \delta_{m n} x_{i} x_{l}+\delta_{i l} \delta_{m n} x_{j} x_{k}+\delta_{j l} \delta_{m n} x_{i} x_{k} \\
& +\delta_{l k} \delta_{m n} x_{i} x_{j}+\delta_{i m} \delta_{n l} x_{j} x_{k}+\delta_{j m} \delta_{n l} x_{i} x_{k}+\delta_{k m} \delta_{n l} x_{i} x_{j}+\delta_{l m} \delta_{k n} x_{i} x_{j}
\end{aligned}
$$




$$
\begin{aligned}
\tilde{G}_{i j k l m n}= & \delta_{i j} x_{k} x_{l} x_{m} x_{n}+\delta_{i k} x_{j} x_{l} x_{m} x_{n}+\delta_{j k} x_{i} x_{l} x_{m} x_{n}+\delta_{i l} x_{j} x_{k} x_{m} x_{n}+\delta_{j l} x_{i} x_{k} x_{m} x_{n}+\delta_{l k} x_{i} x_{j} x_{m} x_{n}+\delta_{i m} x_{j} x_{k} x_{l} x_{n} \\
& +\delta_{j m} x_{i} x_{k} x_{l} x_{n}+\delta_{k m} x_{i} x_{j} x_{l} x_{n}+\delta_{l m} x_{i} x_{j} x_{k} x_{n}+\delta_{i n} x_{j} x_{k} x_{l} x_{m}+\delta_{n j} x_{i} x_{k} x_{l} x_{m}+\delta_{k n} x_{i} x_{j} x_{l} x_{m}+\delta_{n l} x_{i} x_{j} x_{k} x_{m} \\
& +\delta_{m n} x_{i} x_{j} x_{k} x_{l}
\end{aligned}
$$

The average of the third integral and its derivatives in spherical inclusion are:

$$
\begin{aligned}
\langle M(\mathbf{x}, k)\rangle_{\Omega}= & k^{2}+\frac{\Phi(k) \Gamma(k)}{R^{3}} 3 k^{2} \\
\left\langle M_{, i j}(\mathbf{x}, k)\right\rangle_{\Omega}= & \frac{\Phi(k) \Gamma(k)}{R^{3}} \delta_{i j} \\
\left\langle M_{, i j k l}(\mathbf{x}, k)\right\rangle_{\Omega}= & \frac{\Phi(k) \Gamma(k)}{5 R^{3} k^{2}} \tilde{A}_{i j k l}=\frac{\Phi(k) \Gamma(k)}{5 R^{3} k^{2}}\left(\delta_{i j} \delta_{k l}+\delta_{i k} \delta_{j l}+\delta_{j k} \delta_{i l}\right) \\
\left\langle M_{, i j k l m n}(\mathbf{x}, k)\right\rangle_{\Omega}= & \frac{\Phi(k) \Gamma(k)}{35 R^{3} k^{4}} \tilde{E}_{i j k l m n} \\
= & \frac{\Phi(k) \Gamma(k)}{35 R^{3} k^{4}}\left(\delta_{i j} \delta_{k l} \delta_{m n}+\delta_{i k} \delta_{j l} \delta_{m n}+\delta_{j k} \delta_{i l} \delta_{m n}+\delta_{i j} \delta_{k m} \delta_{l n}+\delta_{i j} \delta_{l m} \delta_{k n}+\delta_{i k} \delta_{j m} \delta_{l n}+\delta_{i k} \delta_{l m} \delta_{j n}\right. \\
& \left.+\delta_{j k} \delta_{i m} \delta_{l n}+\delta_{j k} \delta_{l m} \delta_{i n}+\delta_{i l} \delta_{j m} \delta_{k n}+\delta_{i l} \delta_{k m} \delta_{j n}+\delta_{j l} \delta_{i m} \delta_{k n}+\delta_{j l} \delta_{k m} \delta_{n i}+\delta_{l k} \delta_{i m} \delta_{j n}+\delta_{l k} \delta_{j m} \delta_{n i}\right) \\
\left\langle M_{, i}(\mathbf{x}, k)\right\rangle_{\Omega}= & \left\langle M_{, i j k}(\mathbf{x}, k)\right\rangle_{\Omega}=\left\langle M_{, i j k l m}(\mathbf{x}, k)\right\rangle_{\Omega}=0
\end{aligned}
$$

where $\Phi(k)=-k(k+R) e^{-R / k}, \quad \Gamma(k)=R \cosh \left(\frac{R}{k}\right)-k \sinh \left(\frac{R}{k}\right)$

\section{B.2. Cylindrical inclusion}

The analytical expressions of the first two integrals are found in Ma and Gao (2010).

They are:

$$
\begin{aligned}
& \psi(\mathbf{x})=-\frac{1}{32}\left(z^{4}+c_{1} z^{2}+c_{2}\right) \\
& \phi(\mathbf{x})=-\frac{1}{4}\left(z^{2}+c_{3}\right)
\end{aligned}
$$

and

$$
\begin{aligned}
& \psi_{, \alpha \beta \gamma \rho}=-\frac{1}{4}\left(\delta_{\alpha \beta} \delta_{\gamma \rho}+\delta_{\alpha \gamma} \delta_{\beta \rho}+\delta_{\beta \gamma} \delta_{\alpha \rho}\right) \\
& \phi_{, \alpha \beta}=-\frac{1}{2} \delta_{\alpha \beta}
\end{aligned}
$$

where $z=\sqrt{x_{1}^{2}+x_{2}^{2}}$ and $c_{1}, c_{2}, c_{3}$ are constants whose values are of no interest here since only the derivatives of $\psi(\mathbf{x})$ and $\phi(\mathbf{x})$ are involved in the expressions of Eshelby tensors (Ma \& Gao, 2010).

The analytical expressions of the third integral in Eq. (3.13) for a cylindrical inclusion are given by Cheng and He (1997) and Ma and Gao (2010):

$$
M(\mathbf{x}, k)=k^{2}+\Phi(k) \mathrm{I}_{0}\left(\frac{z}{k}\right)
$$

where $\Phi(k)=-k R \mathrm{~K}_{1}\left(\frac{R}{k}\right), \mathrm{I}_{0}\left(\frac{z}{k}\right)$ and $\mathrm{K}_{1}\left(\frac{R}{k}\right)$ are 0 order and 1 order modified Bessel functions of the first kind and second kind respectively.

By defining $N(z, k)=M(\mathbf{x}, k)$ and $D=\frac{1}{z} \frac{d}{d z}$, there are:

$$
D^{l} N=\frac{\Phi(k)}{(k z)^{l}} \mathrm{I}_{l}\left(\frac{z}{k}\right), \quad l \geq 1, \quad \text { no summation for } l
$$

then:

$$
\begin{aligned}
M_{, 3}(\mathbf{x}, k) & =0 \\
M_{, \alpha}(\mathbf{x}, k) & =x_{\alpha} D N \\
M_{, \alpha \beta}(\mathbf{x}, k) & =\delta_{\alpha \beta} D N+x_{\alpha} x_{\beta} D^{2} N \\
M_{, \alpha \beta \gamma}(\mathbf{x}, k) & =\left(\delta_{\alpha \beta} x_{\gamma}+\delta_{\alpha \gamma} x_{\beta}+\delta_{\beta \gamma} x_{\alpha}\right) D^{2} N+x_{\alpha} x_{\beta} x_{\gamma} D^{3} N \\
M_{, \alpha \beta \gamma \rho}(\mathbf{x}, k) & =\hat{A}_{\alpha \beta \gamma \rho} D^{2} N+\hat{B}_{\alpha \beta \gamma \rho} D^{3} N+x_{\alpha} x_{\beta} x_{\gamma} x_{\rho} D^{4} N \\
M_{, \alpha \beta \gamma \rho \xi}(\mathbf{x}, k) & =\hat{C}_{\alpha \beta \gamma \rho \xi} D^{3} N+\hat{D}_{\alpha \beta \gamma \rho \xi} D^{4} N+x_{\alpha} x_{\beta} x_{\gamma} x_{\rho} x_{\xi} D^{5} N
\end{aligned}
$$




$$
M_{, \alpha \beta \gamma \rho \xi \zeta}(\mathbf{x}, k)=\hat{E}_{\alpha \beta \gamma \rho \xi \zeta} D^{3} N+\hat{F}_{\alpha \beta \gamma \rho \xi \zeta} D^{4} N+\hat{G}_{\alpha \beta \gamma \rho \xi \zeta} D^{5} N+x_{\alpha} x_{\beta} x_{\gamma} x_{\rho} x_{\xi} x_{\zeta} D^{6} N
$$

where:

$$
\begin{aligned}
& \hat{A}_{\alpha \beta \gamma \rho}=\delta_{\alpha \beta} \delta_{\gamma \rho}+\delta_{\alpha \gamma} \delta_{\beta \rho}+\delta_{\beta \gamma} \delta_{\alpha \rho} \\
& \hat{B}_{\alpha \beta \gamma \rho}=\delta_{\alpha \beta} x_{\gamma} x_{\rho}+\delta_{\alpha \gamma} x_{\beta} x_{\rho}+\delta_{\beta \gamma} x_{\alpha} x_{\rho}+\delta_{\alpha \rho} x_{\gamma} x_{\beta}+\delta_{\beta \rho} x_{\alpha} x_{\gamma}+\delta_{\rho \gamma} x_{\alpha} x_{\beta} \\
& \hat{C}_{\alpha \beta \gamma \rho \xi}=\delta_{\alpha \beta} \delta_{\gamma \rho} x_{\xi}+\delta_{\alpha \gamma} \delta_{\beta \rho} x_{\xi}+\delta_{\beta \gamma} \delta_{\alpha \rho} x_{\xi}+\delta_{\alpha \beta} \delta_{\gamma \xi} x_{\rho}+\delta_{\alpha \beta} \delta_{\rho \xi} x_{\gamma}+\delta_{\alpha \gamma} \delta_{\beta \xi} x_{\rho}+\delta_{\alpha \gamma} \delta_{\rho \xi} x_{\beta}+\delta_{\beta \gamma} \delta_{\alpha \xi} x_{\rho} \\
& +\delta_{\beta \gamma} \delta_{\xi \rho} x_{\alpha}+\delta_{\alpha \rho} \delta_{\gamma \xi} x_{\beta}+\delta_{\alpha \rho} \delta_{\beta \xi} x_{\gamma}+\delta_{\beta \rho} \delta_{\alpha \xi} x_{\gamma}+\delta_{\beta \rho} \delta_{\gamma \xi} x_{\alpha}+\delta_{\rho \gamma} \delta_{\alpha \xi} x_{\beta}+\delta_{\rho \gamma} \delta_{\beta \xi} x_{\alpha} \\
& \hat{D}_{\alpha \beta \gamma \rho \xi}=\delta_{\alpha \beta} x_{\gamma} x_{\rho} x_{\xi}+\delta_{\alpha \gamma} x_{\beta} x_{\rho} x_{\xi}+\delta_{\beta \gamma} x_{\alpha} x_{\rho} x_{\xi}+\delta_{\alpha \rho} x_{\gamma} x_{\beta} x_{\xi}+\delta_{\beta \rho} x_{\alpha} x_{\gamma} x_{\xi}+\delta_{\rho \gamma} x_{\alpha} x_{\beta} x_{\xi} \\
& +\delta_{\alpha \xi} x_{\beta} x_{\gamma} x_{\rho}+\delta_{\beta \xi} x_{\alpha} x_{\gamma} x_{\rho}+\delta_{\gamma \xi} x_{\alpha} x_{\beta} x_{\rho}+\delta_{\rho \xi} x_{\alpha} x_{\beta} x_{\gamma} \\
& \hat{E}_{\alpha \beta \gamma \rho \xi \zeta}=\delta_{\alpha \beta} \delta_{\gamma \rho} \delta_{\xi \zeta}+\delta_{\alpha \gamma} \delta_{\beta \rho} \delta_{\xi \zeta}+\delta_{\beta \gamma} \delta_{\alpha \rho} \delta_{\xi \zeta}+\delta_{\alpha \beta} \delta_{\gamma \xi} \delta_{\rho_{\zeta}}+\delta_{\alpha \beta} \delta_{\rho \xi} \delta_{\gamma \zeta}+\delta_{\alpha \gamma} \delta_{\beta \xi} \delta_{{ }_{S}}+\delta_{\alpha \gamma} \delta_{\rho \xi} \delta_{\beta \zeta} \\
& +\delta_{\beta \gamma} \delta_{\alpha \xi} \delta_{\rho \varsigma}+\delta_{\beta \gamma} \delta_{\xi \rho} \delta_{\alpha \varsigma}+\delta_{\alpha \rho} \delta_{\gamma \xi} \delta_{\beta \varsigma}+\delta_{\alpha \rho} \delta_{\beta \xi} \delta_{\gamma \varsigma}+\delta_{\beta \rho} \delta_{\alpha \xi} \delta_{\gamma \varsigma}+\delta_{\beta \rho} \delta_{\gamma \xi} \delta_{\alpha \varsigma}+\delta_{\rho \gamma} \delta_{\alpha \xi} \delta_{\beta \varsigma} \\
& +\delta_{\rho \gamma} \delta_{\beta \xi} \delta_{\alpha \varsigma} \\
& \hat{F}_{\alpha \beta \gamma \rho \xi \zeta}=\delta_{\alpha \beta} \delta_{\gamma \rho} x_{\xi} x_{\zeta}+\delta_{\alpha \gamma} \delta_{\beta \rho} x_{\xi} x_{\zeta}+\delta_{\beta \gamma} \delta_{\alpha \rho} x_{\xi} x_{\zeta}+\delta_{\alpha \beta} \delta_{\gamma \xi} x_{\rho} x_{\zeta}+\delta_{\alpha \beta} \delta_{\rho \xi} x_{\gamma} x_{\zeta}+\delta_{\alpha \gamma} \delta_{\beta \xi} x_{\rho} x_{\zeta} \\
& +\delta_{\alpha \gamma} \delta_{\rho \xi} x_{\beta} x_{\zeta}+\delta_{\beta \gamma} \delta_{\alpha \xi} x_{\rho} x_{\zeta}+\delta_{\beta \gamma} \delta_{\xi \rho} x_{\alpha} x_{\zeta}+\delta_{\alpha \rho} \delta_{\gamma \xi} x_{\beta} x_{\zeta}+\delta_{\alpha \rho} \delta_{\beta \xi} x_{\gamma} x_{\zeta}+\delta_{\beta \rho} \delta_{\alpha \xi} x_{\gamma} x_{\zeta} \\
& +\delta_{\beta \rho} \delta_{\gamma \xi} x_{\alpha} x_{\zeta}+\delta_{\rho \gamma} \delta_{\alpha \xi} x_{\beta} x_{\zeta}+\delta_{\rho \gamma} \delta_{\beta \xi} x_{\alpha} x_{\zeta}+\delta_{\alpha \beta} \delta_{\gamma \varsigma} x_{\rho} x_{\xi}+\delta_{\alpha \beta} \delta_{\rho \zeta} x_{\gamma} x_{\xi}+\delta_{\alpha \beta} \delta_{\xi \zeta} x_{\gamma} x_{\rho} \\
& +\delta_{\alpha \gamma} \delta_{\beta \varsigma} x_{\rho} x_{\xi}+\delta_{\alpha \gamma} \delta_{\rho \varsigma} x_{\beta} x_{\xi}+\delta_{\alpha \gamma} \delta_{\xi_{\zeta}} x_{\beta} x_{\rho}+\delta_{\beta \gamma} \delta_{\alpha \varsigma} x_{\rho} x_{\xi}+\delta_{\beta \gamma} \delta_{\rho \varsigma} x_{\alpha} x_{\xi}+\delta_{\beta \gamma} \delta_{\xi \zeta} x_{\alpha} x_{\rho} \\
& +\delta_{\alpha \rho} \delta_{\gamma \varsigma} x_{\beta} x_{\xi}+\delta_{\alpha \rho} \delta_{\beta \varsigma} x_{\gamma} x_{\xi}+\delta_{\alpha \rho} \delta_{\xi \zeta} x_{\gamma} x_{\beta}+\delta_{\beta \rho} \delta_{\alpha \varsigma} x_{\gamma} x_{\xi}+\delta_{\beta \rho} \delta_{\gamma \varsigma} x_{\alpha} x_{\xi}+\delta_{\beta \rho} \delta_{\xi \zeta} x_{\alpha} x_{\gamma} \\
& +\delta_{\rho \gamma} \delta_{\alpha \varsigma} x_{\beta} x_{\xi}+\delta_{\rho \gamma} \delta_{\beta \varsigma} x_{\alpha} x_{\xi}+\delta_{\rho \gamma} \delta_{\xi \varsigma} x_{\alpha} x_{\beta}+\delta_{\alpha \xi} \delta_{\beta \varsigma} x_{\gamma} x_{\rho}+\delta_{\alpha \xi} \delta_{\gamma \varsigma} x_{\beta} x_{\rho}+\delta_{\alpha \xi} \delta_{\rho \varsigma} x_{\beta} x_{\gamma} \\
& +\delta_{\beta \xi} \delta_{\alpha \varsigma} x_{\gamma} x_{\rho}+\delta_{\beta \xi} \delta_{\gamma \varsigma} x_{\alpha} x_{\rho}+\delta_{\beta \xi} \delta_{\rho \varsigma} x_{\alpha} x_{\gamma}+\delta_{\gamma \xi} \delta_{\alpha \varsigma} x_{\beta} x_{\rho}+\delta_{\gamma \xi} \delta_{\beta \zeta} x_{\alpha} x_{\rho}+\delta_{\gamma \xi} \delta_{\rho_{\varsigma}} x_{\alpha} x_{\beta} \\
& +\delta_{\rho \xi} \delta_{\alpha \varsigma} x_{\beta} x_{\gamma}+\delta_{\rho \xi} \delta_{\beta \varsigma} x_{\alpha} x_{\gamma}+\delta_{\rho \xi} \delta_{\gamma \varsigma} x_{\alpha} x_{\beta} \\
& \hat{G}_{\alpha \beta \gamma \rho \xi \zeta}=\delta_{\alpha \beta} x_{\gamma} x_{\rho} x_{\xi} x_{\varsigma}+\delta_{\alpha \gamma} x_{\beta} x_{\rho} x_{\xi} x_{\varsigma}+\delta_{\beta \gamma} x_{\alpha} x_{\rho} x_{\xi} x_{\varsigma}+\delta_{\alpha \rho} x_{\gamma} x_{\beta} x_{\xi} x_{\varsigma}+\delta_{\beta \rho} x_{\alpha} x_{\gamma} x_{\xi} x_{\varsigma}+\delta_{\rho \gamma} x_{\alpha} x_{\beta} x_{\xi} x_{\zeta} \\
& +\delta_{\alpha \xi} x_{\beta} x_{\gamma} x_{\rho} x_{\zeta}+\delta_{\beta \xi} x_{\alpha} x_{\gamma} x_{\rho} x_{\zeta}+\delta_{\gamma \xi} x_{\alpha} x_{\beta} x_{\rho} x_{\zeta}+\delta_{\rho \xi} x_{\alpha} x_{\beta} x_{\gamma} x_{\zeta}+\delta_{\alpha \varsigma} x_{\beta} x_{\gamma} x_{\rho} x_{\xi}+\delta_{\beta \zeta} x_{\alpha} x_{\gamma} x_{\rho} x_{\xi} \\
& +\delta_{\gamma \varsigma} x_{\alpha} x_{\beta} x_{\rho} x_{\xi}+\delta_{\rho \varsigma} x_{\alpha} x_{\beta} x_{\gamma} x_{\xi}+\delta_{\xi \varsigma} x_{\alpha} x_{\beta} x_{\gamma} x_{\rho}
\end{aligned}
$$

The average of the third integral and its derivatives in cylindrical inclusion are:

$$
\begin{aligned}
\langle M(\mathbf{x}, k)\rangle_{\Omega}=k^{2}-2 & k^{2} \mathrm{~K}_{1}\left(\frac{R}{k}\right) \mathrm{I}_{1}\left(\frac{R}{k}\right) \\
\left\langle M_{, \alpha \beta}(\mathbf{x}, k)\right\rangle_{\Omega}=- & \delta_{\alpha \beta} \mathrm{K}_{1}\left(\frac{R}{k}\right) \mathrm{I}_{1}\left(\frac{R}{k}\right) \\
\left\langle M_{, \alpha \beta \gamma \rho}(\mathbf{x}, k)\right\rangle_{\Omega}= & -\frac{1}{4 k^{2}} \mathrm{~K}_{1}\left(\frac{R}{k}\right) \mathrm{I}_{1}\left(\frac{R}{k}\right) \hat{A}_{\alpha \beta \gamma \rho}=-\frac{1}{4 k^{2}} \mathrm{~K}_{1}\left(\frac{R}{k}\right) \mathrm{I}_{1}\left(\frac{R}{k}\right)\left(\delta_{\alpha \beta} \delta_{\gamma \rho}+\delta_{\alpha \gamma} \delta_{\beta \rho}+\delta_{\alpha \rho} \delta_{\beta \gamma}\right) \\
\left\langle M_{, \alpha \beta \gamma \rho \xi \zeta}(\mathbf{x}, k)\right\rangle_{\Omega}= & -\frac{1}{24 k^{4}} \mathrm{~K}_{1}\left(\frac{a}{k}\right) \mathrm{I}_{1}\left(\frac{a}{k}\right) \hat{E}_{\alpha \beta \gamma \rho \xi \zeta} \\
= & -\frac{1}{24 k^{4}} \mathrm{~K}_{1}\left(\frac{a}{k}\right) \mathrm{I}_{1}\left(\frac{a}{k}\right)\left(\delta_{\alpha \beta} \delta_{\gamma \rho} \delta_{\xi \zeta}+\delta_{\alpha \gamma} \delta_{\beta \rho} \delta_{\xi \zeta}+\delta_{\beta \gamma} \delta_{\alpha \rho} \delta_{\xi \zeta}+\delta_{\alpha \beta} \delta_{\gamma \xi} \delta_{\rho \varsigma}+\delta_{\alpha \beta} \delta_{\rho \xi} \delta_{\gamma \varsigma}\right. \\
& +\delta_{\alpha \gamma} \delta_{\beta \xi} \delta_{\rho \varsigma}+\delta_{\alpha \gamma} \delta_{\rho \xi} \delta_{\beta \varsigma}+\delta_{\beta \gamma} \delta_{\alpha \xi} \delta_{\rho \varsigma}+\delta_{\beta \gamma} \delta_{\xi \rho} \delta_{\alpha \zeta}+\delta_{\alpha \rho} \delta_{\gamma \xi} \delta_{\beta \varsigma}+\delta_{\alpha \rho} \delta_{\beta \xi} \delta_{\gamma \zeta} \\
& \left.+\delta_{\beta \rho} \delta_{\alpha \xi} \delta_{\gamma \varsigma}+\delta_{\beta \rho} \delta_{\gamma \xi} \delta_{\alpha \zeta}+\delta_{\rho \gamma} \delta_{\alpha \xi} \delta_{\beta \varsigma}+\delta_{\rho \gamma} \delta_{\beta \xi} \delta_{\alpha \zeta}\right) \\
\left\langle M_{, \alpha}(\mathbf{x}, k)\right\rangle_{\Omega}= & \left\langle M_{, \alpha \beta \gamma}(\mathbf{x}, k)\right\rangle_{\Omega}=\left\langle M_{, \alpha \beta \gamma \rho \xi}(\mathbf{x}, k)\right\rangle_{\Omega}=0
\end{aligned}
$$

B.3. Ellipsoidal inclusion

$a$ and $b$ are defined as the half short axis and half major axis of an ellipsoid respectively. And the major axis lines with axis $x_{3}$.

Because only the derivatives of $\psi(\mathbf{x})$ and $\phi(\mathbf{x})$ are involved in expressions of Eshelby tensors, the expressions of the two integrals are too complicated to be listed here. Their derivatives are (Mura, 1987):

$$
\begin{aligned}
& \psi_{, i j k l}=-\delta_{i j} \delta_{k l}\left[I_{k}-a_{I}^{2} I_{I K}\right]-\left(\delta_{i k} \delta_{j l}+\delta_{j k} \delta_{i l}\right)\left[I_{J}-a_{I}^{2} I_{I J}\right] \\
& \phi_{, i j}=-\delta_{i j} I_{I}
\end{aligned}
$$

where

$$
I_{1}=I_{2}=\frac{g}{2}, \quad I_{3}=1-g, \quad a^{2} I_{13}=a^{2} I_{23}=\frac{3 g-2}{2\left(\rho^{2}-1\right)}, \quad b^{2} I_{33}=\frac{1}{3}-\frac{\rho^{2}(3 g-2)}{3\left(\rho^{2}-1\right)}
$$




$$
\begin{aligned}
& a^{2} I_{11}=a^{2} I_{22}=a^{2} I_{12}=\frac{1}{4}-\frac{3 g-2}{8\left(\rho^{2}-1\right)} \\
& \rho>1, \quad g=\frac{\rho}{\left(\rho^{2}-1\right)^{3 / 2}}\left[\rho \sqrt{\rho^{2}-1}-\cosh ^{-1}(\rho)\right] \\
& \rho<1, \quad g=\frac{\rho}{\left(1-\rho^{2}\right)^{3 / 2}}\left[\cosh ^{-1}(\rho)-\rho \sqrt{1-\rho^{2}}\right]
\end{aligned}
$$

In these formulas, the repeated lowercase indices are summed up from 1 to 3 , the uppercase indices take on the same numbers as the corresponding lowercase ones but are not summed. And $a_{1}=a_{2}=a, \quad a_{3}=b, \quad \rho=b / a$.

According to Ma and Hu (2006), the third integral can be simplified as:

$$
M(\mathbf{x}, k)=\frac{1}{4 \pi} \int_{\Omega} \frac{e^{-r / k}}{r} d \mathbf{x}^{\prime}=k^{2}-k^{2} \frac{b}{2} \int_{0}^{\infty}[p(k) \cdot A(\mathbf{x}, k)] d u
$$

where: $p(k)=\frac{1}{\left(u+b^{2}\right)^{3 / 2}}\left(1+\frac{a}{k} \sqrt{\frac{u+b^{2}}{u+a^{2}}}\right) \exp \left(-\frac{a}{k} \sqrt{\frac{u+b^{2}}{u+a^{2}}}\right), u=b^{2} \tan ^{2} \theta$

$$
\begin{aligned}
& A(\mathbf{x}, k)=I_{0}(B q) \cosh \left(C x_{3}\right) \\
& q=\sqrt{x_{1}^{2}+x_{2}^{2}}, B=\frac{1}{k} \sqrt{\frac{u}{u+a^{2}}}, C=\frac{a}{k \sqrt{u+a^{2}}}
\end{aligned}
$$

$I_{M}$ is the Mth order modified Bessel function of the first kind.

Its derivatives are (Ma \& Hu, 2006):

$$
\begin{gathered}
M_{, i}(\mathbf{x}, k)=k^{2}-k^{2} \frac{b}{2} \int_{0}^{\infty}\left[p(k) \cdot A_{, i}(\mathbf{x}, k)\right] d u \\
M_{, i j}(\mathbf{x}, k)=k^{2}-k^{2} \frac{b}{2} \int_{0}^{\infty}\left[p(k) \cdot A_{, i j}(\mathbf{x}, k)\right] d u \\
M_{, i j k}(\mathbf{x}, k)=k^{2}-k^{2} \frac{b}{2} \int_{0}^{\infty}\left[p(k) \cdot A_{, i j k}(\mathbf{x}, k)\right] d u \\
M_{, i j k l}(\mathbf{x}, k)=k^{2}-k^{2} \frac{b}{2} \int_{0}^{\infty}\left[p(k) \cdot A_{, i j k l}(\mathbf{x}, k)\right] d u \\
M_{, i j k l m}(\mathbf{x}, k)=k^{2}-k^{2} \frac{b}{2} \int_{0}^{\infty}\left[p(k) \cdot A_{, i j k l m}(\mathbf{x}, k)\right] d u \\
M_{, i j k l m n}(\mathbf{x}, k)=k^{2}-k^{2} \frac{b}{2} \int_{0}^{\infty}\left[p(k) \cdot A_{, i j k l m n}(\mathbf{x}, k)\right] d u
\end{gathered}
$$

where

$$
\begin{aligned}
A_{, \alpha}(\mathbf{x}, k) & =x_{\alpha} D N \\
A_{, \alpha \beta}(\mathbf{x}, k) & =\delta_{\alpha \beta} D N+x_{\alpha} x_{\beta} D^{2} N \\
A_{, \alpha \beta \gamma}(\mathbf{x}, k) & =\left(\delta_{\alpha \beta} x_{\gamma}+\delta_{\alpha \gamma} x_{\beta}+\delta_{\beta \gamma} x_{\alpha}\right) D^{2} N+x_{\alpha} x_{\beta} x_{\gamma} D^{3} N \\
A_{, \alpha \beta \gamma \rho}(\mathbf{x}, k) & =\hat{A}_{\alpha \beta \gamma \rho} D^{2} N+\hat{B}_{\alpha \beta \gamma \rho} D^{3} N+x_{\alpha} x_{\beta} x_{\gamma} x_{\rho} D^{4} N \\
A_{, \alpha \beta \gamma \rho \xi}(\mathbf{x}, k) & =\hat{C}_{\alpha \beta \gamma \rho \xi} D^{3} N+\hat{D}_{\alpha \beta \gamma \rho \xi} D^{4} N+x_{\alpha} x_{\beta} x_{\gamma} x_{\rho} x_{\xi} D^{5} N \\
A_{, \alpha \beta \gamma \rho \xi \zeta}(\mathbf{x}, k) & =\hat{E}_{\alpha \beta \gamma \rho \xi \zeta} D^{3} N+\hat{F}_{\alpha \beta \gamma \rho \xi \zeta} D^{4} N+\hat{G}_{\alpha \beta \gamma \rho \xi \zeta} D^{5} N+x_{\alpha} x_{\beta} x_{\gamma} x_{\rho} x_{\xi} x_{\zeta} D^{6} N
\end{aligned}
$$

The Greek letters range from 1 to 2 , and the expressions of $\hat{A}_{\alpha \beta \gamma \rho}, \hat{B}_{\alpha \beta \gamma \rho}, \hat{C}_{\alpha \beta \gamma \rho \xi}, \hat{D}_{\alpha \beta \gamma \rho \xi}, \hat{E}_{\alpha \beta \gamma \rho \xi \zeta}, \hat{F}_{\alpha \beta \gamma \rho \xi \varsigma}, \hat{G}_{\alpha \beta \gamma \rho \xi \varsigma}$ are the same as (B.21).

$$
\begin{aligned}
& D=\frac{1}{q} \frac{d}{d q}, N(q)=A(\mathbf{x}, k)=I_{0}(B q) \cosh \left(C x_{3}\right), \\
& D^{l} N=\cosh \left(C x_{3}\right)\left(\frac{B}{q}\right)^{l} I_{l}(B q), \quad l \geq 1, \quad l \text { is an integer and no summation here }
\end{aligned}
$$

and

$$
A_{, 3}=C \sinh \left(C x_{3}\right) I_{0}(B q)
$$




$$
\begin{aligned}
A_{, 33} & =C^{2} \cosh \left(C x_{3}\right) I_{0}(B q), A_{, \alpha 3}=\left(A_{, \alpha}\right)_{, 3} \\
A_{, 333} & =C^{3} \sinh \left(C x_{3}\right) I_{0}(B q), A_{, \alpha 33}=\left(A_{, \alpha}\right)_{, 33}, A_{, \alpha \beta 3}=\left(A_{, \alpha \beta}\right)_{, 3} \\
A_{, 3333} & =C^{4} \cosh \left(C x_{3}\right) I_{0}(B q), A_{, \alpha 333}=\left(A_{, \alpha}\right)_{, 333}, A_{, \alpha \beta 33}=\left(A_{, \alpha \beta}\right)_{, 33}, \\
A_{, \alpha \beta \gamma 3} & =\left(A_{, \alpha \beta \gamma}\right)_{, 3} \\
A_{, 33333} & =C^{5} \sinh \left(C x_{3}\right) I_{0}(B q), A_{, \alpha 3333}=\left(A_{, \alpha}\right)_{, 3333}, A_{, \alpha \beta 333}=\left(A_{, \alpha \beta}\right)_{, 333}, \\
A_{, \alpha \beta \gamma 33} & =\left(A_{, \alpha \beta \gamma}\right)_{, 33}, A_{, \alpha \beta \gamma \rho 3}=\left(A_{, \alpha \beta \gamma \rho}\right)_{, 3} \\
A_{, 333333} & =C^{6} \cosh \left(C x_{3}\right) I_{0}(B q), A_{, \alpha 33333}=\left(A_{, \alpha}\right)_{, 33333}, A_{, \alpha \beta 3333}=\left(A_{, \alpha \beta}\right)_{, 3333}, \\
A_{, \alpha \beta \gamma 333} & =\left(A_{, \alpha \beta \gamma}\right)_{, 333}, A_{, \alpha \beta \gamma \rho 33}=\left(A_{, \alpha \beta \gamma \rho}\right)_{, 33}, A_{, \alpha \beta \gamma \rho \xi 3}=\left(A_{, \alpha \beta \gamma \rho \xi}\right)_{, 3}
\end{aligned}
$$

\section{References}

Altan, B. S., \& Aifantis, E. C. (1997). On some aspects in the special theory of gradient elasticity. Journal of the Mechanical Behavior of Materials, 8, $231-282$. Buechner, P. M., \& Lakes, R. S. (2003). Size effects in the elasticity and viscoelasticity of bone. Biomechanics and Modeling in Mechanobiology, 1(4), 295-301. https://doi.org/10.1007/s10237-002-0026-8.

Chawla, N., Jones, J. W., Andres, C., \& Allison, J. E. (1998). Effect of SiC volume fraction and particle size on the fatigue resistance of a $2080 \mathrm{Al} / \mathrm{SiC} \mathrm{p}$ composite. Metallurgical and Materials Transactions A, 29(11), 2843-2854. https://doi.org/10.1007/s11661-998-0325-5.

Chen, Y., Lee, J. D., \& Eskandarian, A. (2004). Atomistic viewpoint of the applicability of microcontinuum theories. International Journal of Solids and Structures, 41(8), 2085-2097. https://doi.org/10.1016/j.ijsolstr.2003.11.030.

Cheng, Z. Q., \& He, L. H. (1995). Micropolar elastic fields due to a spherical inclusion. International Journal of Engineering Science, 33(3), 389-397.

Cheng, Z. Q., \& He, L. Q. (1997). Micropolar elastic fields due to a circular cylindrical inclusion. International Journal of Engineering Science, 35(7), 659-668.

El-Daly, A. A., Abdelhameed, M., Hashish, M., \& Eid, A. M. (2012). Synthesis of Al/SiC nanocomposite and evaluation of its mechanical properties using pulse echo overlap method. Journal of Alloys and Compounds, 542, 51-58. https://doi.org/10.1016/j.jallcom.2012.07.102.

Eringen, A. C. (1968). Theory of micropolar elasticity. In H. Liebowitz (Ed.), Fracture, 1 (pp. 621-729). NY: Academic Press.

Eringen, A. C. (1999). Microcontinuum field theories I: foundations and solids. New York: Springer-Verlag.

Fleck, N. A., Muller, G. M., Ashby, M. F., \& Hutchinson, J. W. (1994). Strain gradient plasticity: Theory and experiment. Acta Metallurgica Et Materialia. https://doi.org/10.1016/0956-7151(94)90502-9.

Forest, S., \& Sievert, R. (2003). Elastoviscoplastic constitutive frameworks for generalized continua. Acta Mechanica, 160(1-2), 71-111. https://doi.org/10.1007/ s00707-002-0975-0.

Gao, X. L., \& Ma, H. M. (2009). Green's function and Eshelby's tensor based on a simplified strain gradient elasticity theory. Acta Mechanica, 207(3-4), 163-181. https://doi.org/10.1007/s00707-008-0109-4.

Gao, X. L., \& Ma, H. M. (2010). Strain gradient solution for Eshelby’s ellipsoidal inclusion problem. Proceedings of the Royal Society A: Mathematical, Physical and Engineering Sciences, 466(February), 2425-2446. https://doi.org/10.1098/rspa.2009.0631.

Gao, X. L., \& Park, S. K. (2007). Variational formulation of a simplified strain gradient elasticity theory and its application to a pressurized thick-walled cylinder problem. International Journal of Solids and Structures, 44, 7486-7499.

Germain, P. (1973). The method of virtual power in continuum mechanics. Part 2: Microstructure. SIAM Journal of Applied Mathematics, 25(3), 556-575.

Hu, G., Liu, X., \& Lu, T. J. (2005). A variational method for non-linear micropolar composites. Mechanics of Materials, 37(4), 407-425. https://doi.org/10.1016/ j.mechmat.2004.03.006.

Kleinert, H. (1989). Gauge fields in condensed matter: 2. World Scientific.

Koiter, W. T. (1964). Couple stresses in the theory of elasticity, I and II. Nederlandse Akademie Van Wetenschappen Series B, 67, 17-44.

Kouzeli, M., \& Mortensen, A. (2002). Size dependent strengthening in particle reinforced aluminium. Acta Materialia, 50, 39-51.

Liu, X., \& Hu, G. (2005). A continuum micromechanical theory of overall plasticity for particulate composites including particle size effect. International Journal of Plasticity, 21(4), 777-799. https://doi.org/10.1016/j.ijplas.2004.04.014.

Llorca, J., Suresh, S., \& Needleman, A. (1992). An experimental and numerical study of cyclic deformation in metal-matrix composites. Metallurgical Transactions A, 23(3), 919-934. https://doi.org/10.1007/BF02675568.

Ma, H., \& Hu, G. (2006). Eshelby tensors for an ellipsoidal inclusion in a micropolar material. International Journal of Engineering Science, 44, 595-605. https://doi.org/10.1016/j.ijsolstr.2006.09.003.

Ma, H. M., \& Gao, X. L. (2010). Eshelby's tensors for plane strain and cylindrical inclusions based on a simplified strain gradient elasticity theory. Acta Mechanica, 211(1-2), 115-129. https://doi.org/10.1007/s00707-009-0221-0.

Ma, H. M., \& Gao, X. L. (2014). A new homogenization method based on a simplified strain gradient elasticity theory. Acta Mechanica, 225(4-5), 1075-1091. https://doi.org/10.1007/s00707-013-1059-z.

Mindlin, R. D. (1964). Micro-structure in linear elasticity. Archive for Rational Mechanics and Analysis, 16(1), 51-78. https://doi.org/10.1007/BF00248490.

Mindlin, R. D. (1965). Second gradient of strain and surface-tension in linear elasticity. International Journal of Solids and Structures, 1, 417-438.

Mindlin, R. D., \& Tiersten, H. F. (1962). Effects of couple-stresses in linear elasticity. Archive for Rational Mechanics and Analysis, 11(1), 415-448. https: //doi.org/10.1007/BF00253946.

Mura, T. (1987). Micromechanics of defects in solids (2nd edn). Dordrecht: Martinus Nijhoff.

Sandru, N. (1966). On some problems of the linear theory of the asymmetric elasticity. International Journal of Engineering Science, 4, 81-96.

Shaat, M., \& Abdelkefi, A. (2016). On a second-order rotation gradient theory for linear elastic continua. International Journal of Engineering Science, 100, 74-98. https://doi.org/10.1016/j.ijengsci.2015.11.009.

Sharma, P., \& Dasgupta, A. (2002). Average elastic field and scale-dependent overall properties of heterogeneous micropolar materials containing spherical and cylindrical inhomogeneities. Physical Review B, 66(224110), 1-10.

Song, J., Liu, J., Ma, H., Liang, L., \& Wei, Y. (2014). Determinations of both length scale and surface elastic parameters for fcc metals. Comptes Rendus Mecanique, 342(5), 315-325. https://doi.org/10.1016/j.crme.2014.03.004.

Toupin, R. A. (1962). Elastic materials with couple-stresses. Archive for Rational Mechanics and Analysis, 11(1), 385-414. https://doi.org/10.1007/BF00253945.

Wang, Y. M., Zhang, C. H., Zong, Y. P., \& Yang, H. P. (2013). Experimental and simulation studies of particle size effects on tensile deformation behavior of iron matrix composites. Advanced Composite Materials, 22(5), 299-310. https://doi.org/10.1080/09243046.2013.812550.

Wei, Y. (2006). A new finite element method for strain gradient theories and applications to fracture analyses. European Journal of Mechanics, A/Solids, 25(6), 897-913. https://doi.org/10.1016/j.euromechsol.2006.03.001.

Wei, Y., \& Hutchinson, J. (1997). Steady-state crack growth and work of fracture for solids characterized by strain gradient plasticity. Journal of the Mechanics and Physics of Solids, 45(8), 1253-1273.

Xun, F., Hu, G., \& Huang, Z. (2004). Effective in plane moduli of composites with a micropolar matrix and coated fibers. International Journal of Solids and Structures, 41(1), 247-265. https://doi.org/10.1016/j.ijsolstr.2003.09.018. 
Zhang, X., \& Sharma, P. (2005). Inclusions and inhomogeneities in strain gradient elasticity with couple stresses and related problems. International Journal of Solids and Structures, 42(13), 3833-3851. https://doi.org/10.1016/j.ijsolstr.2004.12.005.

Zheng, Q., \& Zhao, Z.-H. (2004). Green's Function and Eshelby's Fields in Couple-Stress Elasticity. International Journal for Multiscale Computational Engineering, 2(1), 15-27. 